\title{
Generalized impedance boundary conditions for strongly absorbing obstacle: The full wave equation
}

\author{
Hoai-Minh Nguyen \\ Department of Mathematics, \\ École Polytechnique Fédérale de Lausanne, \\ EPFL SB MATHAA CAMA, Station 8, \\ CH-1015 Lausanne, Switzerland \\ hoai-minh.nguyen@epfl.ch \\ Linh Viet Nguyen \\ Department of Mathematics, University of Idaho, \\ 875 Perimeter Drive MS 441103, \\ Moscow, ID 83843, USA \\ lnguyen@uidaho.edu
}

Received 23 April 2014

Revised 10 March 2015

Accepted 18 March 2015

Published 4 June 2015

Communicated by C. Conca

This paper is devoted to the study of the generalized impedance boundary conditions (GIBCs) for a strongly absorbing obstacle in the time regime in two and three dimensions. The GIBCs in the time domain are heuristically derived from the corresponding conditions in the time harmonic regime. The latter is frequency-dependent except the one of order 0 ; hence the formers are non-local in time in general. The error estimates in the time regime can be derived from the ones in the time harmonic regime when the frequency dependence is well controlled. This idea is originally due to Nguyen and Vogelius [Approximate cloaking for the full wave equation via change of variables, SIAM J. Math. Anal. 44 (2012) 769-807] for the cloaking context. In this paper, we present the analysis to the GIBCs of orders 0 and 1. To implement the ideas in [H.-M. Nguyen and M. S. Vogelius, Approximate cloaking for the full wave equation via change of variables, SIAM J. Math. Anal. 44 (2012) 769-807], we revise and extend the work of Haddar, Joly, and Nguyen, [Generalized impedance boundary condition for scattering by strongly absorbing obstacles: the scalar case, Math. Models Methods Appl. Sci. 15 (2005) 12731300], where the GIBCs were investigated for a fixed frequency in three dimensions. Even though we heavily follow the strategy in [H.-M. Nguyen and M. S. Vogelius, Approximate cloaking for the full wave equation via change of variables, SIAM J. Math. Anal. 44 (2012) 769-807], our analysis on the stability contains new ingredients and ideas. First, instead of considering the difference between solutions of the exact model and the approximate model, we consider the difference between their derivatives in time. This 


\begin{abstract}
simple idea helps us to avoid the machinery used in [H.-M. Nguyen and M. S. Vogelius, Approximate cloaking for the full wave equation via change of variables, SIAM J. Math. Anal. 44 (2012) 769-807] concerning the integrability with respect to frequency in the low frequency regime. Second, in the high frequency regime, the Morawetz multiplier technique used in [H.-M. Nguyen and M. S. Vogelius, Approximate cloaking for the full wave equation via change of variable, SIAM J. Math. Anal. 44 (2012) 769-807] does not fit directly in our setting. Our proof makes use of a result by Hörmander in $\left[L^{p}\right.$ estimates for (pluri-) subharmonic functions, Math. Scand. 20 (1967) 65-78]. Another important part of the analysis in this paper is the well-posedness in the time domain for the approximate problems imposed with GIBCs on the boundary of the obstacle, which are non-local in time.
\end{abstract}

Keywords: Impedance boundary condition; highly absorbing; wave equation; non-local boundary condition.

AMS Subject Classification: 35L05, 65M15, 35Q60, 35A01, 35A02, 35B35

\title{
1. Introduction and Statement of the Main Results
}

The computation of electromagnetic scattering from an arbitrary obstacle has been an active research area for many decades. One technique is to replace the exact model inside the obstacle by appropriate boundary conditions on its surface; hence the problem of determining the external electromagnetic fields can be solved without considering the fields inside the obstacle (see, e.g. Refs. 14 and 30). These boundary conditions are called Generalized Impedance Boundary Conditions (GIBCs). The first GIBC for a highly absorbing obstacle (highly conducting obstacle) was proposed by Leontovich (see, e.g. Ref. 17) and was extended later by Rytov. ${ }^{29}$ Other main contributors to this problem include Shchukin, ${ }^{31}$ Grünberg, ${ }^{11}$ and Feiberg, ${ }^{9}$ just to name a few. Antoine, Barucq, and Vernhet, ${ }^{1}$ using the technique of pseudodifferential operators (following the ideas of Engquist and Majda ${ }^{8}$ ), implemented a new derivation of such conditions. Recently, Haddar, Joly, and Nguyen ${ }^{12}$ revisited these GIBCs for the Helmholtz equation. More precisely, the authors first proposed a new construction of GIBCs which is based on an ansatz for the asymptotic expansion of exact solutions. They then developed mathematical tools, based on compactness arguments, to establish error estimates up to order 3. Related works are the eddy current problem studied by MacCamy and Stephan ${ }^{18}$ and the study of the GIBCs for highly conducting obstacle for the Maxwell system in the time harmonic regime by Haddar, Joly, and $\operatorname{Nguyen}^{13}$ (see also the work of Caloz, Dauge, Faou, and Péron ${ }^{6}$ ) and references therein.

The study of GIBCs for highly absorbing obstacle has been though studied extensively in the literature, the rigorous study of GIBCs for highly absorbing obstacle in the time regime is not known to our knowledge. The lack of the study in the time regime is not special for this context but a common problem in the study of acoustic and electromagnetic waves since problems in the time regime involve the interaction of all frequencies and hence they are harder to analyze.

The goal of this paper is to provide the analysis of the GIBCs for highly absorbing obstacle in the time regime in two and three dimensions. Heuristically, these 
are obtained by taking the inverse Fourier transform of the corresponding conditions in the time harmonic regime with respect to frequency. Since the GIBCs in the time harmonic regime are frequency-dependent, the ones in the time regime are non-local with respect to time. The error estimates in the time regime can be derived from the ones in the time harmonic regime, when the frequency dependence is well controlled. This idea is originally due to Nguyen and Vogelius ${ }^{23}$ used for the cloaking context. To implement this idea, we revise and extend the work of Haddar, Joly, and Nguyen, ${ }^{12}$ where the GIBCs for the time harmonic regime were investigated for a fixed frequency in three dimensions. Even though we follow the strategy in Ref. 23, our analysis on the stability contains new ingredients and ideas. First, instead of considering the difference between solutions of the exact model and the approximate model in the time harmonic regime, we consider the difference between their derivatives in time. This simple idea helps us to avoid the machinery used in Ref. 23 concerning the integrability with respect to frequency in the low frequency regime. The proof of the stability in the low frequency regime is based on a compactness argument as in Ref. 12 and uses ideas in Ref. 20. Second, the compactness argument is not appropriate in the high frequency regime; moreover, the well-known Morawetz multiplier technique used in Ref. 23 does not fit directly in our setting, mainly due to the structure of the GIBCs. This new technical challenge distinguishes our work from Ref. 23. To tackle it, we use essentially a result by Hörmander ${ }^{15}$ (see Lemma 5.3 in this paper). Another important part of the proof is the well-posedness in the time domain of the approximate problems imposed with GIBCs on the boundary of the obstacle, which are non-local in time.

In this paper, we concentrate only on the GIBCs of order 0 and 1 . Even though our approach also works for the GIBCs of orders 2 and 3, we are still not able to obtain the optimal expected estimates as in the time harmonic regime due to the complexity of the structures of the GIBCs in these cases. We postpone the study of these conditions to our future work. ${ }^{25}$

There are many other interesting situations in which the asymptotic expansions have been investigated in the time harmonic setting. For example, the thin coating effect (e.g. Ref. 4), the wave propagation in media with thin slots (e.g. Ref. 16), the wave propagation across thin periodic interfaces (e.g. Ref. 7) and references therein. We hope that our analysis also sheds light to the situations mentioned.

We next describe the problem in more details. Let $\Omega$ be a smooth bounded domain in $\mathbb{R}^{d}(d=2,3)$ with boundary $\Gamma:=\partial \Omega$ and let $f \in L^{2}\left(\mathbb{R}_{+} \times \mathbb{R}^{d}\right)$ be such that supp $f \subset[0, T] \times\left(B_{R_{0}} \backslash \bar{\Omega}\right)$ for some fixed $R_{0}>0$ and $T>0$.

Here and in what follows, $B_{r}$ denotes the ball of radius $r$ centered at the origin. Let $u^{\epsilon} \in L_{\text {loc }}^{\infty}\left([0, \infty) ; H^{1}\left(\mathbb{R}^{d}\right)\right)$ with $\partial_{t} u^{\epsilon} \in L_{\text {loc }}^{\infty}\left([0, \infty) ; L^{2}\left(\mathbb{R}^{d}\right)\right)$ be the unique weak solution to the problem

$$
\begin{cases}\partial_{t t} u^{\epsilon}(t, x)-\Delta u^{\epsilon}(t, x)+\sigma_{\epsilon}(x) \partial_{t} u^{\epsilon}(t, x)=f(t, x), & (t, x) \in \mathbb{R}_{+} \times \mathbb{R}^{d}, \\ u^{\epsilon}(0, x)=\partial_{t} u^{\epsilon}(0, x)=0, & x \in \mathbb{R}^{d},\end{cases}
$$


where

$$
\sigma_{\epsilon}(x)= \begin{cases}0 & \text { if } x \in \mathbb{R}^{d} \backslash \Omega, \\ \frac{1}{\epsilon^{2}} & \text { if } x \in \Omega,\end{cases}
$$

for some $\epsilon>0$ small. Roughly speaking, the absorption of $\Omega$ is of order $1 / \epsilon^{2}$. We consider here the case in which the initial conditions are zero. The general case could be treated similarly as discussed in the cloaking setting in Ref. 23 .

Let $\hat{u}_{\epsilon}(k, x)$ and $\hat{f}(k, x)$ be the Fourier transform of $u_{\epsilon}$ and $f$ with respect to time respectively, i.e. ${ }^{\mathrm{a}}$ :

$$
\hat{u}_{\epsilon}(k, x)=\mathcal{F}\left(u_{\epsilon}\right)(k, x)=\frac{1}{\sqrt{2 \pi}} \int_{\mathbb{R}} u_{\epsilon}(t, x) e^{i k t} d t
$$

and

$$
\hat{f}(k, x)=\mathcal{F}(f)(k, x)=\frac{1}{\sqrt{2 \pi}} \int_{\mathbb{R}} f(t, x) e^{i k t} d t .
$$

Then for almost every $k>0, \hat{u}_{\epsilon}(k, x) \in H_{\mathrm{loc}}^{1}\left(\mathbb{R}^{d}\right)$ be the unique solution to the equation

$$
\Delta \hat{u}_{\epsilon}(k, x)+k^{2} \hat{u}_{\epsilon}(k, x)+i k \sigma_{\epsilon} \hat{u}_{\epsilon}(k, x)=\hat{f}(k, x), \quad \text { in } \mathbb{R}^{d},
$$

which satisfies the outgoing condition

$$
\partial_{r} u_{\epsilon}-i k u_{\epsilon}=o\left(r^{-\frac{(d-1)}{2}}\right), \quad \text { as } r=|x| \rightarrow \infty .
$$

This fact is formulated later in Lemma 2.4 whose proof has root in Ref. 23 (Theorem A.1).

Set $^{\mathrm{b}}$ :

$$
\hat{\epsilon}=\frac{\epsilon}{\sqrt{k}}, \quad \alpha=\frac{\sqrt{2}}{2}-i \frac{\sqrt{2}}{2},
$$

and

$$
\mathcal{D}_{\ell}^{\hat{\epsilon}}= \begin{cases}0 & \text { for } \ell=0, \\ \frac{\hat{\epsilon}}{\alpha} & \text { for } \ell=1 .\end{cases}
$$

It is proved in Ref. 12 that the GIBCs of order 0 and 1 corresponding to (1.4) are

$$
v+\mathcal{D}_{\ell}^{\hat{\epsilon}} \partial_{n} v=0, \quad \text { on } \Gamma \text {. }
$$

Here and in what follows, $n=n(x)$ is the unit normal vector directed into $\Omega$ on $\Gamma$. More precisely, let $\mathbf{s} \in H^{1}\left(\mathbb{R}^{d}\right)$ with support in $B_{R_{0}} \backslash \Omega$ and let $v^{\epsilon} \in H_{\text {loc }}^{1}\left(\mathbb{R}^{d}\right)$ and

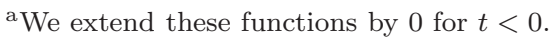

${ }^{\mathrm{b}} \alpha^{2}=-i$. 
$v_{\ell}^{a} \in H_{\mathrm{loc}}^{1}\left(\mathbb{R}^{d} \backslash \Omega\right)$ be the unique outgoing solutions to the problems

$$
\Delta v^{\epsilon}+k^{2} v^{\epsilon}+i k \sigma_{\epsilon} v^{\epsilon}=\mathbf{s}, \quad \text { in } \mathbb{R}^{d},
$$

and

$$
\begin{cases}\Delta v_{\ell}^{a}+k^{2} v_{\ell}^{a}=\mathbf{s}, & \text { in } \mathbb{R}^{d} \backslash \Omega, \\ v_{\ell}^{a}+\mathcal{D}_{\ell}^{\hat{\epsilon}} \partial_{n} v_{\ell}^{a}=0 & \text { on } \Gamma .\end{cases}
$$

Haddar, Joly, and Nguyen (see Theorem 3 in Ref. 12) proved that, for any $R>0{ }^{\text {c }}$

$$
\left\|v^{\epsilon}-v_{\ell}^{a}\right\|_{H^{1}\left(B_{R} \backslash \Omega\right)} \leq C(k, R) \epsilon^{\ell+1}\|\mathbf{s}\|_{H^{m}\left(\mathbb{R}^{d}\right)},
$$

for some positive constant $C(k, R)$ and for some $m>0$ large enough. The dependence on $k$ of $C(k, R)$ in Ref. 12 is not explicit.

We are now ready to heuristically derive the GIBCs for (1.1) by taking the inverse Fourier transform of the GIBCs in the time harmonic regime with respect to frequency. We have

GIBC of order 0 :

$$
G_{0}^{\epsilon}(v):=v=0, \quad \text { on } \mathbb{R}_{+} \times \Gamma .
$$

This is clear from (1.7) and (1.8) with $\ell=0$.

GIBC of order 1:

$$
G_{1}^{\epsilon}(v):=\partial_{n} v+B_{1}^{\epsilon} v=0 \quad \text { on } \mathbb{R}_{+} \times \Gamma,
$$

where

$$
\left(B_{1}^{\epsilon} v\right)(t, x):=\frac{1}{\sqrt{\pi} \epsilon} \int_{0}^{t} \frac{\partial_{t} v(\tau, x)}{\sqrt{t-\tau}} d \tau .
$$

The derivation goes as follows. For $\ell=1$, condition (1.8) reads as:

$$
\partial_{n} v+\frac{\alpha \sqrt{k}}{\epsilon} v=0, \quad \text { on } \Gamma,
$$

or equivalently

$$
\partial_{n} v+\frac{1}{\epsilon} \frac{1}{\alpha \sqrt{k}}(-i k) v=0, \quad \text { on } \Gamma .
$$

The condition (1.13) is now a consequence of the fact (see, e.g. p. 171 in Ref. 10):

$$
\mathcal{F}(\varphi)(k)=\frac{1}{\sqrt{2 \pi}} \frac{\sqrt{\pi}}{\alpha \sqrt{k}}, \quad \text { where } \quad \varphi(t)= \begin{cases}\frac{1}{\sqrt{t}} & \text { if } t>0, \\ 0 & \text { if } t \leq 0 .\end{cases}
$$

We have heuristically derived the GIBCs of orders 0 (1.12) and 1 (1.13) for the full wave equation (1.1). Similarly, one can obtain the GIBCs of orders 2, 3

${ }^{\mathrm{c}}$ In Ref. 12, the authors considered the bounded setting. However, their method also implies the results mentioned here. 
for (1.1) from the corresponding ones in the time harmonic regime obtained in Ref. 12. However, such conditions are more complicated. We have not been able yet to obtain the optimal expected estimates for them as in the ones in the time harmonic regime. We postpone the study of these conditions to our future work. ${ }^{25}$

The goal of this paper is to establish error estimates for (1.12) and (1.13). More precisely, we prove the following theorem.

Theorem 1.1. Let $d=2,3, \ell=0,1, T>0, R_{0}>0$, and let $G_{\ell}^{\epsilon}$ be defined in (1.12) and (1.13). Assume that $f \in L^{2}\left([0, \infty) \times \mathbb{R}^{d}\right)$ with $\operatorname{supp} f \subset[0, T] \times\left(B_{R_{0}} \backslash \bar{\Omega}\right)$. There exists a unique weak solution $u_{\ell}^{a} \in L_{\mathrm{loc}}^{\infty}\left([0, \infty) ; H^{1}\left(\mathbb{R}^{d} \backslash \Omega\right)\right)$ with $\partial_{t} u_{\ell}^{a} \in$ $L_{\text {loc }}^{\infty}\left([0, \infty) ; L^{2}\left(\mathbb{R}^{d} \backslash \Omega\right)\right)$ to

$$
\begin{cases}\partial_{t t}^{2} u_{\ell}^{a}-\Delta u_{\ell}^{a}=f & \text { in } \mathbb{R}_{+} \times \mathbb{R}^{d} \backslash \bar{\Omega} \\ G_{\ell}^{\epsilon}\left(u_{\ell}^{a}\right)=0 & \text { on } \mathbb{R}_{+} \times \Gamma \\ \partial_{t} u_{\ell}^{a}(0, \cdot)=u_{\ell}^{a}(0, \cdot)=0 & \text { in } \mathbb{R}^{d} \backslash \bar{\Omega}\end{cases}
$$

moreover,

$$
\int_{\mathbb{R}^{d} \backslash \Omega}\left|\nabla u_{\ell}^{a}(t, x)\right|^{2}+\left|\partial_{t} u_{\ell}^{a}(t, x)\right|^{2} d x \leq C t\|f\|_{L^{2}\left([0, t] \times \mathbb{R}^{d}\right)}^{2} \quad \forall t \geq 0 .
$$

Assume in addition that $\Omega$ is star-shaped and $f \in C^{\infty}\left((0, \infty) \times \mathbb{R}^{d}\right)$ with $\operatorname{supp} f \subset \subset$ $(0, T] \times\left(B_{R_{0}} \backslash \bar{\Omega}\right)$. Then, for any $t>0$ and $K \subset \subset \mathbb{R}^{d} \backslash \bar{\Omega},{ }^{\mathrm{d}}$ there is a positive constant $C$ independent of $\epsilon$ and $f$, such that, for some integer $m^{\mathrm{e}}$ :

$$
\left\|u^{\epsilon}-u_{\ell}^{a}\right\|_{L^{\infty}\left([0, t] ; H^{1}(K)\right)} \leq C \epsilon^{\ell+1}\|f\|_{H^{m}\left(\mathbb{R}_{+} \times \mathbb{R}^{d}\right)} .
$$

The following definition of the weak solutions, which is motivated from the standard concept of weak solutions, is used in Theorem 1.1.

Definition 1.1. Let $d=2,3$ and $\ell=0,1$. We say a function

$$
u_{\ell}^{a} \in L_{\mathrm{loc}}^{\infty}\left([0, \infty) ; H^{1}\left(\mathbb{R}^{d} \backslash \Omega\right)\right) \quad \text { with } \quad \partial_{t} u_{\ell}^{a} \in L_{\mathrm{loc}}^{\infty}\left([0, \infty) ; L^{2}\left(\mathbb{R}^{d} \backslash \Omega\right)\right)
$$

is a weak solution to (1.17) provided

$$
\begin{aligned}
& \frac{d^{2}}{d t^{2}} \int_{\mathbb{R}^{d} \backslash \Omega} u_{\ell}^{a}(t, x) v(x) d x+\int_{\mathbb{R}^{d} \backslash \Omega} \nabla u_{\ell}^{a}(t, x) \nabla v(x) d x+\ell \int_{\Gamma}\left(B_{\ell}^{\epsilon} u_{\ell}^{a}\right)(t, x) v(x) d x \\
& \quad=\int_{\mathbb{R}^{d} \backslash \Omega} f(t, x) v(x) d x \quad \forall v \in H^{1}\left(\mathbb{R}^{d} \backslash \Omega\right)
\end{aligned}
$$

for any $t>0$, and

$$
u_{\ell}^{a}(0, x)=\partial_{t} u_{\ell}^{a}(0, x)=0 \quad \text { in } \mathbb{R}^{d} \backslash \Omega
$$

\footnotetext{
${ }^{\mathrm{d}}$ Roughly speaking, $K$ is bounded and away from the boundary of the obstacle.

e The integer $m$ can be chosen as follows: $m=13$ if $\ell=0$ and $m=16$ if $\ell=1$. Assume in addition that supp $f \cap \bar{\Omega}=\emptyset$. Then $m$ can be chosen as follows: $m=8$ if $\ell=0$ and $m=9$ if $\ell=1$; however the constant $C$ in (1.19) now depends on the distance between supp $f$ and $\bar{\Omega}$ (see footnote i).
} 
Remark 1.1. In the definition, the last term on the left-hand side of (1.20) is 0 if $\ell=0$. The definition in the case $\ell=0$ is standard.

The proof of Theorem 1.1 is presented in Sec. 2. The proof of well-posedness of (1.17) for $\ell=1$ (non-local structure in time) is based on a non-trivial energy estimate (1.18), which is derived from the causality, see (1.14). Following the strategy in Ref. 23, we will derive (1.19) from estimates in the frequency domain. For this end, we establish estimates for $C(k, R)$ in $(1.11)$ where the dependence on $k$ is well controlled. This is one of the main parts of the analysis and presented in the following three propositions which deal with different regimes of frequency.

Proposition 1.1. Let $d=2,3, \ell=0,1,0<\epsilon<1,0<k<\epsilon^{2}, R_{0}>0$, and $\mathbf{s} \in L^{2}\left(\mathbb{R}^{d} \backslash \bar{\Omega}\right)$ with supp $\mathbf{s} \subset B_{R_{0}} \backslash \bar{\Omega}$. Let $v^{\epsilon} \in H_{\mathrm{loc}}^{1}\left(\mathbb{R}^{d}\right)$ and $v_{\ell}^{a} \in H_{\mathrm{loc}}^{1}\left(\mathbb{R}^{d} \backslash \Omega\right)$ be the unique outgoing solutions to (1.9) and (1.10) respectively. We have, for any $r>0$,

$$
\left\|v^{\epsilon}-v_{\ell}^{a}\right\|_{H^{1}\left(B_{r} \backslash \Omega\right)} \leq C_{r} \hat{\epsilon}^{2}\|\mathbf{s}\|_{L^{2}\left(\mathbb{R}^{d}\right)},
$$

for some constant $C_{r}>0$, independent of $\mathbf{s}, \epsilon$ and $k$.

Proposition 1.2. Let $d=2,3, \ell=0,1,0<\epsilon<1, k_{0}>0, \epsilon^{2}<k<k_{0}, R_{0}>0$, and $\mathbf{s} \in H^{2 \ell+5}\left(\mathbb{R}^{d} \backslash \bar{\Omega}\right)$ with supp $\mathbf{s} \subset B_{R_{0}} \backslash \bar{\Omega}$. Let $v^{\epsilon} \in H_{\mathrm{loc}}^{1}\left(\mathbb{R}^{d}\right)$ and $v_{\ell}^{a} \in H_{\mathrm{loc}}^{1}\left(\mathbb{R}^{d} \backslash \Omega\right)$ be the unique outgoing solutions to (1.9) and (1.10) respectively. We have, for any $r>0$,

$$
\left\|v^{\epsilon}-v_{\ell}^{a}\right\|_{H^{1}\left(B_{r} \backslash \Omega\right)} \leq C_{r} \hat{\epsilon}^{\ell+1}\|\mathbf{s}\|_{H^{2 \ell+5}\left(\mathbb{R}^{d}\right)},
$$

for some constant $C_{r}>0$, independent of $\mathbf{s}, \epsilon$ and $k$.

We recall here that $\hat{\epsilon}$ is given in (1.6).

Proposition 1.3. Let $d=2,3, \ell=0,1, k_{0}>0,0<\epsilon<1, k \geq k_{0}, R_{0}>0$, and $\mathbf{s} \in H^{2 \ell+5}\left(\mathbb{R}^{d} \backslash \bar{\Omega}\right)$ with supp $\mathbf{s} \subset B_{R_{0}} \backslash \bar{\Omega}$. Let $v^{\epsilon} \in H_{\mathrm{loc}}^{1}\left(\mathbb{R}^{d}\right)$ and $v_{\ell}^{a} \in H_{\mathrm{loc}}^{1}\left(\mathbb{R}^{d} \backslash \Omega\right)$ be the unique outgoing solutions to (1.9) and (1.10) respectively. Assume that $\Omega$ is star-shaped. Then, for any $K \subset \subset \mathbb{R}^{d} \backslash \bar{\Omega}$, we have

$$
\left\|\nabla\left(v^{\epsilon}-v_{\ell}^{a}\right)\right\|_{L^{2}(K)}+k\left\|v^{\epsilon}-v_{\ell}^{a}\right\|_{L^{2}(K)} \leq C_{K} k^{2 \ell+7} \hat{\epsilon}^{\ell+1}\|\mathbf{s}\|_{H^{2 \ell+5}\left(\mathbb{R}^{d}\right)},
$$

for some constant $C_{K}>0$, independent of $\mathbf{s}, \epsilon$ and $k$.

The proofs of Propositions 1.1 and 1.2 are given in Sec. 4. They are based on a compactness argument as in Ref. 12 and use results on the Helmholtz equations in the low frequency regime in Refs. 20 and 21. The proof of Proposition 1.2 and 1.3 uses the asymptotic expansion introduced in Ref. 12. To obtain explicit dependence on frequency of these estimates, we revise the asymptotic expansion given in Ref. 12 for all range of frequency with a focus on the dependence on frequency. The proof of the stability in Proposition 1.3 is given in Sec. 5. It is a heart matter of our paper. The compactness argument used in the proof of Propositions 1.1 and 1.2 is not appropriate in this regime. Moreover, the Morawetz's multiplier technique does not work directly in our settings. Due to the structure of the GIBCs, we are 
only able to obtain an estimate in $L^{2}(\Gamma)$-norm of the solution, not the $H^{1}(\Gamma)$-norm required for Morawetz's technique. To overcome this difficulty, we use a result due to Hörmander in Ref. 15 (see Lemma 5.3 in this paper). The payoff for lacking of the control of the $H^{1}(\Gamma)$-norm is that we can only obtain estimates in regions away from $\Gamma$, see (1.23).

Remark 1.2. One can verify that $B_{1}^{\epsilon}$ given in the definition of the GIBC of order 1 is of the form $\frac{1}{\epsilon} \partial_{t}^{1 / 2}$, where $\partial_{t}^{1 / 2}$ is the fractional derivative of order $1 / 2$ with respect to time. The operator $\partial_{t}^{1 / 2}$ also appears in the absorbing boundary conditions for the Schrödinger equation, see, e.g. Refs. 2 and 3. The micro-local analysis technique was used to obtain the absorbing boundary conditions for the Schrödinger equation. ${ }^{2,3}$ It would be interesting to see whether the micro-local analysis technique is suitable for the high frequency case considered in this paper, where another scale $\epsilon$ exists. On the other hand, it would be also interesting to know whether our analysis can be used to establish the boundary conditions for the Schrödinger equation, at least in the linear case. ${ }^{f}$

\section{Proof of Theorem 1.1}

This section, containing two subsections, is devoted to the proof of Theorem 1.1 assuming Propositions 1.1, 1.2, and 1.3 (their proofs are given in Secs. 4 and 5). In the first subsection, we establish the well-posedness and the stability for (1.17). We also show that the Fourier transform of the weak solution satisfies the outgoing conditions for almost every positive frequency. The proof of Theorem 1.1 is given in the second subsection.

\subsection{Preliminaries}

In this section, we prepare some materials for the proof of Theorem 1.1. We first prove the well-posedness and the stability for (1.17).

Lemma 2.1. Let $d=2,3, \ell=0,1$ and $f \in L^{2}\left([0, \infty), L^{2}\left(\mathbb{R}^{d} \backslash \Omega\right)\right)$ with compact support. There exists a unique weak solution $v_{\ell}^{a} \in L_{\mathrm{loc}}^{\infty}\left([0, \infty), H^{1}\left(\mathbb{R}^{d} \backslash \Omega\right)\right)$ with $\partial_{t} v_{\ell}^{a} \in L_{\text {loc }}^{\infty}\left([0, \infty), L^{2}\left(\mathbb{R}^{d} \backslash \Omega\right)\right)$ to $(1.17)$. Moreover

$$
E\left(t, u_{\ell}^{a}\right) \leq C t\|f\|_{L^{2}\left([0, t] \times \mathbb{R}^{d}\right)}^{2} \quad \forall t \geq 0 .
$$

Here,

$$
E(t, \psi):=\frac{1}{2} \int_{\mathbb{R}^{d} \backslash \Omega}\left(\left|\partial_{t} \psi(t, x)\right|^{2}+|\nabla \psi(t, x)|^{2}\right) d x,
$$

for $\psi \in L_{\mathrm{loc}}^{\infty}\left([0, \infty), H^{1}\left(\mathbb{R}^{d} \backslash \Omega\right)\right)$ with $\partial_{t} \psi \in L_{\mathrm{loc}}^{\infty}\left([0, \infty), L^{2}\left(\mathbb{R}^{d} \backslash \Omega\right)\right)$.

\footnotetext{
${ }^{\mathrm{f}}$ The authors thank the referee for pointing out the connection between this work and the Schrödinger equation.
} 
Proof. We only prove the theorem for $\ell=1$, since the case $\ell=0$ is standard. We first establish the existence of a weak solution which satisfies (2.1). For this end, we use the Galerkin method. Let $\left(\varphi_{j}\right)_{j=1}^{\infty} \subset C_{c}^{\infty}\left(\mathbb{R}^{d} \backslash \Omega\right)$ be an orthonormal basis in $H^{1}\left(\mathbb{R}^{d} \backslash \Omega\right)$. For $m \in \mathbb{N}$, consider $u_{m}$ of the form

$$
u_{m}=\sum_{j=1}^{m} d_{m, j}(t) \varphi_{j}(x)
$$

such that

$$
\begin{aligned}
& \frac{d^{2}}{d t^{2}} \int_{\mathbb{R}^{d} \backslash \Omega} u_{m}(t, x) \varphi_{j}(x) d x+\int_{\mathbb{R}^{d} \backslash \Omega} \nabla u_{m}(t, x) \nabla \varphi_{j}(x) d x \\
& \quad+\int_{\Gamma}\left(B_{1}^{\epsilon} u_{m}\right)(t, x) \varphi_{j}(x) d x=\int_{\mathbb{R}^{d} \backslash \Omega} f(t, x) \varphi_{j}(x) d x, \quad \text { for } j=1, \ldots, m
\end{aligned}
$$

and

$$
d_{m, j}(0)=d_{m, j}^{\prime}(0)=0 \quad \text { for } j=1, \ldots, m .
$$

Since $\left(\varphi_{j}\right)_{j}$ is linearly independent in $H^{1}\left(\mathbb{R}^{d} \backslash \Omega\right)$, it is also linearly independent in $L^{2}\left(\mathbb{R}^{d} \backslash \Omega\right)$. This implies the $(n \times n)$ matrix $M$ given by $M_{i, j}=\left\langle\varphi_{i}, \varphi_{j}\right\rangle_{L^{2}\left(\mathbb{R}^{d} \backslash \Omega\right)}$ is invertible. The existence and uniqueness of $u_{m}$ then follows; for example, one can use the theory of Volterra equation, see, e.g. Ref. 5 (Theorem 2.1.1).

We now derive an estimate for $u_{m}$. Let us multiply $(2.4)$ by $d_{m, j}^{\prime}(s)$ and sum it up with respect to $j$. Integrating the resulting equation over $[0, t]$ with respect to $s$ and using (2.5), we obtain

$$
\begin{gathered}
E\left(t, u_{m}\right)+\int_{0}^{t} \int_{\Gamma}\left(B_{1}^{\epsilon} u_{m}\right)(s, x) \partial_{t} u_{m}(s, x) d x d s \\
=\int_{0}^{t} \int_{\mathbb{R}^{d} \backslash \Omega} f(s, x) \partial_{t} u_{m}(s, x) d x d s .
\end{gathered}
$$

We claim that, for $t \geq 0$,

$$
\int_{0}^{t} \int_{\Gamma}\left(B_{1}^{\epsilon} u_{m}\right)(s, x) \partial_{t} u_{m}(s, x) d x d s \geq 0 .
$$

Indeed, recall

$$
\left(B_{1}^{\epsilon} u_{m}\right)(s, x)=\frac{1}{\sqrt{\pi} \epsilon}\left(\varphi * \partial_{t} u_{m}\right)(s, x)
$$

where $\varphi$ is given in (1.16). Here and in what follows $*$ denotes the convolution with respect to time. Set

$$
U(s, x)= \begin{cases}\partial_{t} u_{m}(s, x) & \text { if } s<t \\ 0 & \text { if } s \geq t \text { or } s<0 .\end{cases}
$$

Then,

$$
\int_{0}^{t} \int_{\Gamma}\left(B_{1}^{\epsilon} u_{m}\right)(s, x) \partial_{t} u_{m}(s, x) d x d s=\frac{1}{\epsilon \sqrt{\pi}} \int_{\mathbb{R}} \int_{\Gamma}(\varphi * U)(s, x) U(s, x) d x d s .
$$


Using Parseval's identity and (1.16), we obtain

$$
\begin{aligned}
\int_{\mathbb{R}} \int_{\Gamma}(\varphi * U)(s, x) U(s, x) d x d s & =\int_{\mathbb{R}} \int_{\Gamma}(\widehat{\varphi * U})(k, x) \widehat{\widehat{U}}(k, x) d x d k \\
& =2 \Re \int_{\mathbb{R}_{+}} \int_{\Gamma} \frac{\sqrt{\pi}}{\alpha \sqrt{k}}|\widehat{U}(k, x)|^{2} d x d k \geq 0 .
\end{aligned}
$$

A combination of (2.8) and (2.9) yields (2.7).

From (2.6) and (2.7), we have

$$
E\left(t, u_{m}\right) \leq \int_{0}^{t} \int_{\mathbb{R}^{d} \backslash \Omega} f(s, x) \partial_{t} u_{m}(s, x) d x d s .
$$

By the Gronwall inequality, it follows from (2.2) and (2.10) that

$$
\int_{0}^{t} E\left(s, u_{m}\right) d s \leq C\left[\int_{0}^{t}\left(\int_{0}^{s} \int_{\mathbb{R}^{d} \backslash \Omega}|f(\tau, x)|^{2} d x d \tau\right)^{1 / 2} d s\right]^{2}
$$

which implies

$$
\int_{0}^{t} E\left(s, u_{m}\right) d s \leq C t^{2} \int_{0}^{t} \int_{\mathbb{R}^{d} \backslash \Omega}|f(s, x)|^{2} d x d s .
$$

From (2.10),

$$
E\left(t, u_{m}\right) \leq\left(\int_{0}^{t} \int_{\mathbb{R}^{d} \backslash \Omega}\left|\partial_{t} u_{m}(s, x)\right|^{2} d x d s\right)^{1 / 2}\left(\int_{0}^{t} \int_{\mathbb{R}^{d} \backslash \Omega}|f(s, x)|^{2} d x d s\right)^{1 / 2} .
$$

This yields

$$
E\left(t, u_{m}\right) \leq\left(\int_{0}^{t} E\left(s, u_{m}\right) d s\right)^{1 / 2}\left(\int_{0}^{t} \int_{\mathbb{R}^{d} \backslash \Omega}|f(s, x)|^{2} d x d s\right)^{1 / 2} .
$$

It follows from (2.11) that

$$
E\left(t, u_{m}\right) \leq C t \int_{0}^{t} \int_{\mathbb{R}^{d} \backslash \Omega}|f(s, x)|^{2} d x d s .
$$

Hence, for any fixed $T>0$, there exists a subsequence of $\left(u_{m}\right)$ (which is also denoted by $u_{m}$ for notational ease) such that $u_{m} \rightarrow u$ weakly star in $L^{\infty}\left([0, T], H^{1}\left(\mathbb{R}^{d} \backslash \Omega\right)\right)$ and $\partial_{t} u_{m} \rightarrow \partial_{t} u$ weakly star in $L^{\infty}\left([0, T], L^{2}\left(\mathbb{R}^{d} \backslash \Omega\right)\right)$. It is clear that $u$ satisfies (1.20) for $t \in(0, T)$ and (1.21).

It remains to show that the limit is unique. It suffices to prove that if $u \in$ $L^{\infty}\left([0, T], H^{1}\left(\mathbb{R}^{d} \backslash \Omega\right)\right)$ with $\partial_{t} u \in L^{\infty}\left([0, T], L^{2}\left(\mathbb{R}^{d} \backslash \Omega\right)\right)$ and $u$ satisfies $(1.20)$ with $f=0$ and (1.21) then $u=0$. 
Set $u^{*}(t, x)=\int_{0}^{t} u(\tau, x) d \tau$. We claim that $u^{*}$ satisfies

$$
\begin{aligned}
& \frac{d^{2}}{d t^{2}} \int_{\mathbb{R}^{d} \backslash \Omega} u^{*}(t, x) v(x) d x+\int_{\mathbb{R}^{d} \backslash \Omega} \nabla u^{*}(t, x) \nabla v(x) d x \\
& \quad+\int_{\Gamma}\left(B_{1}^{\epsilon} u^{*}\right)(t, x) v(x) d x=0, \quad \forall v \in H^{1}\left(\mathbb{R}^{d} \backslash \Omega\right),
\end{aligned}
$$

for any $t>0$, and

$$
u^{*}(0, x)=\partial_{t} u^{*}(0, x)=0 \quad \text { in } \mathbb{R}^{d} \backslash \Omega .
$$

The claim (more precisely, Eq. (2.13)) can be verified by integrating (1.20) with respect to $t$. We basically only need to verify the validity of the boundary term on the left-hand side. This follows from Lemma 2.2 below.

From (2.13), we derive

$$
\begin{gathered}
\int_{\mathbb{R}^{d} \backslash \Omega} \partial_{t t}^{2} u^{*}(t, x) v(x) d x+\int_{\mathbb{R}^{d} \backslash \Omega} \nabla u^{*}(t, x) \nabla v(x) d x \\
\quad+\int_{\Gamma}\left(B_{1}^{\epsilon} u^{*}\right)(t, x) v(x) d x=0, \quad \forall v \in H^{1}\left(\mathbb{R}^{d} \backslash \Omega\right) .
\end{gathered}
$$

Letting $v(x)=u_{t}^{* \mathrm{~g}}$ in $(2.15)$ and integrating in $[0, t]$, we obtain

$$
\begin{aligned}
& \int_{0}^{t} \int_{\mathbb{R}^{d} \backslash \Omega} u_{t t}^{*}(s, x) u_{t}^{*}(s, x) d x d s+\int_{0}^{t} \int_{\mathbb{R}^{d} \backslash \Omega} \nabla u^{*}(s, x) \nabla u_{t}^{*}(s, x) d x d s \\
& \quad+\int_{0}^{t} \int_{\Gamma}\left(B_{1}^{\epsilon} u^{*}\right)(s, x) u_{t}^{*}(s, x) d x d s=0 .
\end{aligned}
$$

By the same argument used to obtain (2.7), we have

$$
\int_{0}^{t} \int_{\Gamma}\left(B_{\ell}^{\epsilon} u^{*}\right)(s, x) u_{t}^{*}(s, x) d x \geq 0 .
$$

It follows from (2.16) that

$$
E\left(t, u^{*}\right) \leq E\left(0, u^{*}\right)=0
$$

Therefore, $u^{*} \equiv 0$ and, hence, $u \equiv 0$. The proof is complete.

Remark 2.1. Similar ideas are taken into account for the proof of the wellposedness of the non-local wave equations in Ref. 24 in which the Drude-Lorentz model is used to capture the dependence of the material on the frequency.

The following lemma which reveals an interesting property of the integral kernel $\varphi$ of $B_{1}^{\epsilon}$ is used in the proof of Lemma 2.1.

g Such a choice of $v(x)$ satisfies $v \in H^{1}\left(\mathbb{R}^{d} \backslash \Omega\right)$. This is the reason why we have to work with $u^{*}$ instead of directly with $u$. 
Lemma 2.2. Assume that $\psi \in L_{\mathrm{loc}}^{\infty}[0, \infty)$. Let

$$
\Psi(t)=\int_{0}^{t} \psi(s) d s .
$$

Then, for any $t>0$,

$$
\int_{0}^{t}(\varphi * \psi)(s) d s=(\varphi * \Psi)(t) .
$$

Here, $\varphi$ is defined in (1.16).

Proof. The proof only involves a change of order of integration and an integration by parts. The details are left to the reader.

Concerning the outgoing condition of $\hat{u}_{\ell}^{a}$ and $\hat{u}^{\epsilon}$, we have the following two results whose proofs are similar to that of Theorem A1 in Ref. 23. The details are left to the reader.

Lemma 2.3. Let $d=2,3, \ell=0,1$ and let $\hat{u}_{\ell}^{a}(k, x)$ be the Fourier transform of $u_{\ell}^{a}(t, x)$ with respect to $t$. Then, for almost every $k>0, \hat{u}_{\ell}^{a}(k, \cdot) \in H_{\mathrm{loc}}^{1}\left(\mathbb{R}^{d} \backslash \Omega\right)$ is the unique outgoing solution to

$$
\begin{cases}\Delta \hat{u}_{\ell}^{a}(k, x)+k^{2} \hat{u}_{\ell}^{a}(k, x)=-\hat{f}(k, x), & \text { in } \mathbb{R}^{d} \backslash \Omega, \\ \hat{u}_{\ell}^{a}(k, x)+\mathcal{D}_{\ell}^{\hat{\epsilon}} \partial_{n} \hat{u}_{\ell}^{a}(k, x)=0 & \text { on } \Gamma .\end{cases}
$$

Moreover,

$$
k \hat{u}_{\ell}^{a}(k, x) \in L_{\mathrm{loc}}^{2}\left(\mathbb{R} \times\left(\mathbb{R}^{d} \backslash \Omega\right)\right) .
$$

Lemma 2.4. Let $d=2,3, \ell=0,1$ and let $\hat{u}^{\epsilon}(k, x)$ be the Fourier transform of $u^{\epsilon}(t, x)$ with respect to $t$. Then, for almost every $k>0, \hat{u}^{\epsilon}(k, \cdot) \in H_{\mathrm{loc}}^{1}\left(\mathbb{R}^{d}\right)$ is the unique outgoing solution to

$$
\Delta \hat{u}^{\epsilon}(k, x)+k^{2} \hat{u}^{\epsilon}(k, x)+i k \sigma_{\epsilon} \hat{u}^{\epsilon}(k, x)=-\hat{f}(k, x), \quad \text { in } \mathbb{R}^{d} .
$$

Moreover,

$$
k \hat{u}^{\epsilon}(k, x) \in L_{\mathrm{loc}}^{2}\left(\mathbb{R} \times \mathbb{R}^{d}\right) .
$$

\subsection{Proof of Theorem 1.1}

The unique existence of $u_{\ell}^{a}$ and (1.18) follow from Lemma 2.1. It only remains to prove (1.19). To this end, we use Propositions 1.1-1.3 (their proofs are given later in Secs. 4 and 5). We follow the strategy in Ref. 23. However, instead of estimating $\left\|u^{\epsilon}-u_{\ell}^{a}\right\|_{L^{2}\left(\mathbb{R}_{+}, H^{1}(K)\right)}$ as in Ref. 23, we estimate $\left\|\partial_{t}\left(u^{\epsilon}-u_{\ell}^{a}\right)\right\|_{L^{2}\left(\mathbb{R}_{+}, H^{1}(K)\right)}$. This simple idea helps us to avoid the technical issue of integrability in low frequency range in Ref. 23, which involves the theory of Gamma-convergence. ${ }^{\mathrm{h}}$ 
Applying Parseval's identity and using the fact that $u^{\epsilon}$ and $u_{\ell}^{a}$ are real, we have

$$
\int_{\mathbb{R}_{+}}\left\|\partial_{t}\left(u^{\epsilon}-u_{\ell}^{a}\right)(t, \cdot)\right\|_{H^{1}(K)}^{2} d t=2 \int_{\mathbb{R}_{+}} k^{2}\left\|\left(\hat{u}^{\epsilon}-\hat{u}_{\ell}^{a}\right)(k, \cdot)\right\|_{H^{1}(K)}^{2} d k .
$$

It is clear that

$$
\begin{aligned}
& \int_{\mathbb{R}_{+}} k^{2}\left\|\left(\hat{u}^{\epsilon}-\hat{u}_{\ell}^{a}\right)(k, \cdot)\right\|_{H^{1}(K)}^{2} d k \\
& \quad=\left(\int_{0}^{\epsilon^{2}}+\int_{\epsilon^{2}}^{1}+\int_{1}^{\infty}\right) k^{2}\left\|\left(\hat{u}^{\epsilon}-\hat{u}_{\ell}^{a}\right)(k, \cdot)\right\|_{H^{1}(K)}^{2} d k .
\end{aligned}
$$

We next estimate the right-hand side of (2.18). We begin with the first term. Applying Proposition 1.1, we have

$$
\begin{aligned}
\int_{0}^{\epsilon^{2}} k^{2}\left\|\left(\hat{u}^{\epsilon}-\hat{u}_{\ell}^{a}\right)(k, \cdot)\right\|_{H^{1}(K)}^{2} d k & \leq C \int_{0}^{\epsilon^{2}} k^{2} \hat{\epsilon}^{4}\|\hat{f}(k, \cdot)\|_{L^{2}\left(\mathbb{R}^{d}\right)}^{2} d k \\
& =C \epsilon^{4} \int_{0}^{\epsilon^{2}}\|\hat{f}(k, \cdot)\|_{L^{2}\left(\mathbb{R}^{d}\right)}^{2} d k .
\end{aligned}
$$

It follows that

$$
\int_{0}^{\epsilon^{2}} k^{2}\left\|\left(\hat{u}^{\epsilon}-\hat{u}_{\ell}^{a}\right)(k, \cdot)\right\|_{H^{1}(K)}^{2} d k \leq C \epsilon^{6} \sup _{k>0}\|\hat{f}(k, \cdot)\|_{L^{2}\left(\mathbb{R}^{d}\right)}^{2} .
$$

Since $f(\cdot, x)$ is supported in $[0, T]$, it follows from the definition of the Fourier transform that

$$
|\hat{f}(k, x)| \leq \frac{1}{\sqrt{2 \pi}} \int_{\mathbb{R}}|f(t, x)| d t \leq C\left(\int_{\mathbb{R}}|f(t, x)|^{2} d t\right)^{1 / 2},
$$

which implies

$$
\|\hat{f}(k, \cdot)\|_{L^{2}\left(\mathbb{R}^{d}\right)}^{2} \leq C \int_{\mathbb{R}^{d}} \int_{\mathbb{R}}|f(t, x)|^{2} d t d x=C\|f\|_{L^{2}\left(\mathbb{R}_{+} \times \mathbb{R}^{d}\right)}^{2} .
$$

A combination of $(2.19)$ and $(2.20)$ yields

$$
\int_{0}^{\epsilon^{2}} k^{2}\left\|\left(\hat{u}^{\epsilon}-\hat{u}_{\ell}^{a}\right)(k, \cdot)\right\|_{H^{1}(K)}^{2} d k \leq C \epsilon^{6}\|f\|_{L^{2}\left(\mathbb{R}_{+} \times \mathbb{R}^{d}\right)}^{2} .
$$

We next estimate the second term of the right-hand side in (2.18). Applying Proposition 1.2, we obtain

$$
\begin{aligned}
& \int_{\epsilon^{2}}^{1} k^{2}\left\|\left(\hat{u}^{\epsilon}-\hat{u}_{\ell}^{a}\right)(k, \cdot)\right\|_{H^{1}(K)}^{2} d k \\
& \quad \leq C \sup _{k>0}\|\hat{f}(k, \cdot)\|_{H^{2 \ell+5}\left(\mathbb{R}^{d}\right)}^{2} \int_{\epsilon^{2}}^{1} k^{2} \hat{\epsilon}^{2(\ell+1)} d k .
\end{aligned}
$$

Similar to $(2.20)$, we have

$$
\sup _{k>0}\|\hat{f}(k, \cdot)\|_{H^{2 \ell+5}\left(\mathbb{R}^{d}\right)} \leq C\|f\|_{H^{2 \ell+5}\left(\mathbb{R}_{+} \times \mathbb{R}^{d}\right)} .
$$


Since $\hat{\epsilon}^{2}=\epsilon^{2} / k$ and $\ell=0,1$, it follows that

$$
\int_{\epsilon^{2}}^{1} k^{2} \hat{\epsilon}^{2(\ell+1)} d k=\epsilon^{2(\ell+1)} \int_{\epsilon^{2}}^{1} k^{1-\ell} d k \leq C \epsilon^{2(\ell+1)} .
$$

A combination of $(2.22)-(2.24)$ yields

$$
\int_{\epsilon^{2}}^{1} k^{2}\left\|\left(\hat{u}^{\epsilon}-\hat{u}_{\ell}^{a}\right)(k, \cdot)\right\|_{H^{1}(K)}^{2} d k \leq C \epsilon^{2(\ell+1)}\|f\|_{H^{2 \ell+5}\left(\mathbb{R}_{+} \times \mathbb{R}^{d}\right)}^{2} .
$$

We now estimate the last term of the right-hand side in (2.18). Applying Proposition 1.3 , we obtain

$$
\begin{aligned}
\int_{1}^{\infty} k^{2}\left\|\left(\hat{u}^{\epsilon}-\hat{u}_{\ell}^{a}\right)(k, \cdot)\right\|_{H^{1}(K)}^{2} d k & \leq \int_{1}^{\infty} k^{2} \hat{\epsilon}^{2(\ell+1)} k^{2(2 \ell+7)}\|\hat{f}(k, \cdot)\|_{H^{2 \ell+5}\left(\mathbb{R}^{d}\right)}^{2} d k \\
& \leq C \epsilon^{2(\ell+1)} \int_{1}^{\infty} k^{3 \ell+15}\|\hat{f}(k, .)\|_{H^{2 \ell+5}\left(\mathbb{R}^{d}\right)}^{2} d k
\end{aligned}
$$

It follows that

$$
\int_{1}^{\infty} k^{2}\left\|\left(\hat{u}^{\epsilon}-\hat{u}_{\ell}^{a}\right)(k, \cdot)\right\|_{H^{1}(K)}^{2} d k \leq C \epsilon^{2(\ell+1)}\|f\|_{H^{m_{\ell}}\left(\mathbb{R}_{+} \times \mathbb{R}^{d}\right)}^{2},
$$

where ${ }^{\mathrm{i}}$ :

$$
m_{\ell}=13 \quad \text { if } \ell=0 \text { and } \quad m_{\ell}=16 \quad \text { if } \ell=1 .
$$

Plugging (2.21), (2.25), and (2.26) into (2.18), we obtain:

$$
\int_{\mathbb{R}} k^{2}\left\|\left(\hat{u}^{\epsilon}-\hat{u}_{\ell}^{a}\right)(k, \cdot)\right\|_{H^{1}(K)}^{2} d k \leq C \epsilon^{2(\ell+1)}\|f\|_{H^{m_{\ell}\left(\mathbb{R}_{+} \times \mathbb{R}^{d}\right)}}^{2} .
$$

A combination of (2.17) and (2.27) yields

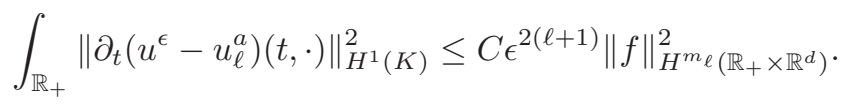

Since $u^{\epsilon}-u_{\ell}^{a} \equiv 0$ at $t=0$, the conclusion follows.

\section{Asymptotic Expansion for Highly Conducting Obstacle Revisited}

This section is on the asymptotic expansion of $v^{\epsilon}$, the solution of (1.9), with respect to the small parameter $\hat{\epsilon}:=\epsilon / \sqrt{k}$ and is essentially based on the work of Ref. 12. Our goal is to keep track of the frequency dependence there. We recall the notations in Ref. 12 and state estimates which are used in the proof of Propositions 1.2 and 1.3. Their proofs are given in the Appendix.

${ }^{\mathrm{i}}$ If supp $f \cap \bar{\Omega}=\emptyset$, then $\|\hat{f}(k, \cdot)\|_{H^{2 \ell+5}\left(\mathbb{R}^{d}\right)}^{2}$ can be replaced by $\|\hat{f}(k, \cdot)\|_{L^{2}\left(\mathbb{R}^{d}\right)}^{2}$. It follows that $m_{\ell}$ can be chosen as follows: $m_{\ell}=8$ if $\ell=0$ and $m_{\ell}=9$ if $\ell=1$ and the constant $C$ in (2.26) now depends on the distance between $\operatorname{supp} f$ and $\bar{\Omega}$. 
Define

$$
\Omega^{\delta}=\{x \in \Omega, d(x, \Gamma) \leq \delta\} .
$$

In what follows, we fix $\delta>0$ small enough such that any $x \in \Omega^{\delta}$ can be written uniquely in the form $x=x_{\Gamma}+\nu n$, where $\left(x_{\Gamma}, \nu\right) \in \Gamma \times \mathbb{R}_{+}$. Here, $n$ is the unit normal vector of $\Gamma$ at $x_{\Gamma}$ pointing toward $\Omega$.

Let $d=3, \mathcal{H}$ and $\mathcal{G}$ be the mean and Gaussian curvatures of $\Gamma$ and let $\mathcal{C}:=\nabla_{\Gamma} n$ be the curvature tensor on $\Gamma$. Define the tangential operator $\mathcal{M}$ by the identity

$$
\mathcal{C M}=G I_{\Gamma} .
$$

One has (see Eq. (4.4) in Ref. 12) for $x \in \Omega^{\delta}$,

$$
J_{\nu}^{3} \Delta=J_{\nu} \operatorname{div}_{\Gamma}\left(I_{\Gamma}+\nu \mathcal{M}\right)^{2} \nabla_{\Gamma}-J_{\nu} \cdot\left(I_{\Gamma}+\nu \mathcal{M}\right)^{2} \nabla_{\Gamma}+J_{\nu}^{3} \partial_{\nu \nu}^{2}+2 J_{\nu}^{2}(\mathcal{H}+\nu G) \partial_{\nu},
$$

where

$$
J_{\nu}:=\operatorname{det}(I+\nu \mathcal{C})=1+2 \nu \mathcal{H}+\nu^{2} G .
$$

The following differential operators $\mathcal{A}_{m}(1 \leq m \leq 8)$ are defined in Ref. $12^{\mathrm{j}}$ :

$$
\begin{aligned}
\mathcal{A}_{1}= & 2 \mathcal{H} \partial_{\eta}+6 \eta \mathcal{H}\left(\partial_{\eta}^{2}+i\right), \\
\mathcal{A}_{2}= & \Delta_{\Gamma}+k^{2}+2 \eta\left(\mathcal{G}+4 \mathcal{H}^{2}\right) \partial_{\eta}+3 \eta^{2}\left(\mathcal{G}+4 \mathcal{H}^{2}\right)\left(\partial_{\eta}^{2}+i\right), \\
\mathcal{A}_{3}= & 2 \eta\left[\mathcal{H} \Delta_{\Gamma}+\operatorname{div}_{\Gamma}\left(\mathcal{M} \nabla_{\Gamma}\right)-\nabla_{\Gamma} \mathcal{H} \nabla_{\Gamma}+3 k^{2} \mathcal{H}\right]+4 \eta^{2} \mathcal{H}\left[\left(3 \mathcal{G}+2 \mathcal{H}^{2}\right) \partial_{\eta}\right] \\
& +4 \eta^{3} \mathcal{H}\left(3 \mathcal{G}+2 \mathcal{H}^{2}\right)\left(\partial_{\eta}^{2}+i\right), \\
\mathcal{A}_{4}= & \eta^{2}\left[\mathcal{G} \Delta_{\Gamma}+4 \mathcal{H} \operatorname{div}_{\Gamma}\left(\mathcal{M} \nabla_{\Gamma}\right)+\operatorname{div}_{\Gamma}\left(\mathcal{M}^{2} \nabla_{\Gamma}\right)\right] \\
& -\eta^{2}\left[\nabla_{\Gamma} \mathcal{G} \nabla_{\Gamma}+4 \nabla_{\Gamma} \mathcal{H}\left(\mathcal{M} \nabla_{\Gamma}\right)-3 k^{2}\left(\mathcal{G}+4 \mathcal{H}^{2}\right)\right] \\
& +4 \eta^{3} \mathcal{G}\left(\mathcal{G}+4 \mathcal{H}^{2}\right) \partial_{\eta}+3 \eta^{4} \mathcal{G}\left(\mathcal{G}+4 \mathcal{H}^{2}\right)\left(\partial_{\eta}^{2}+i\right), \\
\mathcal{A}_{5}= & 2 \eta^{3}\left[\mathcal{G} \operatorname{div}_{\Gamma}\left(\mathcal{M} \nabla_{\Gamma}\right)+\mathcal{H} \operatorname{div}_{\Gamma}\right]-2 \eta^{3}\left[\nabla_{\Gamma} \mathcal{G}\left(\mathcal{M} \nabla_{\Gamma}\right)+\nabla_{\Gamma} \mathcal{H}\left(\mathcal{M}^{2} \nabla_{\Gamma}\right)\right. \\
& \left.-2 k^{2} \mathcal{H}\left(3 \mathcal{G}+2 \mathcal{H}^{2}\right)\right]+10 \eta^{4} \mathcal{G}^{2} \mathcal{H} \partial_{\eta}+6 \eta^{5} \mathcal{G}^{2} \mathcal{H}\left(\partial_{\eta}^{2}+i\right), \\
\mathcal{A}_{6}= & \eta^{4}\left[\mathcal{G} \operatorname{div}_{\Gamma}\left(\mathcal{M}^{2} \nabla_{\Gamma}\right)-\nabla_{\Gamma} \mathcal{G}\left(\mathcal{M}^{2} \nabla_{\Gamma}\right)+3 k^{2} \mathcal{G}\left(\mathcal{G}+4 \mathcal{H}^{2}\right)\right] \\
& +2 \eta^{5} \mathcal{G}^{3} \partial_{\eta}+\eta^{6} \mathcal{G}^{3}\left(\partial_{\eta}^{2}+i\right), \\
\mathcal{A}_{7}= & 6 \eta^{5} k^{2} \mathcal{G}^{3} \mathcal{H}, \quad \text { and } \mathcal{A}_{8}=\eta^{6} k^{2} \mathcal{G}^{3} .
\end{aligned}
$$

Using (3.2) and (3.3), as in Eq. (5.22) of Ref. 12, one has

$$
\Delta+k^{2}+\frac{i}{\hat{\epsilon}^{2}}=\frac{1}{J_{\nu}^{3} \hat{\epsilon}^{2}}\left(-\partial_{\eta \eta}^{2}-i-\sum_{m=1}^{8} \hat{\epsilon}^{m} \mathcal{A}_{m}\right) \text {. }
$$

jThe signs in front of $i$ in our formulae are opposite to the ones in Ref. 12. This is due to the difference between Eq. (1.9) in this paper and Eq. (2.3) in Ref. 12, because of different ways to take the Fourier transform in these papers. The reader should keep this fact in mind when comparing our calculations with those in Ref. 12 . 
Similarly, we also define the above operations in the case $d=2$. In this case, the triple $(\mathcal{H}, \mathcal{G}, \mathcal{M})$ is replaced by $\left(\frac{\kappa}{2}, 0,0\right)$, where $\kappa=\kappa(x)$ is the (signed) curvature of $\Gamma$.

The following definitions by recurrence of $w_{e}^{\ell}$ in $\mathbb{R}^{d} \backslash \Omega$ and $w_{i}^{\ell}$ in $\Gamma \times \mathbb{R}_{+}$are given in Ref. 12. For $\ell=0$, define

$$
w_{i}^{0}(x)=0 \quad \text { in } \Gamma \times \mathbb{R}_{+},
$$

and let $w_{e}^{0} \in H_{\text {loc }}^{1}\left(\mathbb{R}^{d} \backslash \Omega\right)$ be the unique outgoing solution to

$$
\begin{cases}\Delta w_{e}^{0}+k^{2} w_{e}^{0}=\mathbf{s} & \text { in } \mathbb{R}^{d} \backslash \bar{\Omega} \\ w_{e}^{0}=0 & \text { on } \Gamma .\end{cases}
$$

Let $\ell \geq 1$. Assume that $w_{e}^{j}$ and $w_{i}^{j}$ are defined for $j \leq \ell-1$. Define $w_{i}^{\ell}$ to be the solution to

$$
\left\{\begin{array}{l}
\left(\partial_{\eta}^{2}+i\right) w_{i}^{\ell}\left(x_{\Gamma}, \eta\right)=-\sum_{m=1}^{8} \mathcal{A}_{m} w_{i}^{\ell-m}\left(x_{\Gamma}, \eta\right) \quad \text { for }\left(x_{\Gamma}, \eta\right) \in \Gamma \times \mathbb{R}_{+}, \\
\partial_{\eta} w_{i}^{\ell}\left(x_{\Gamma}, 0\right)=\partial_{n} w_{e}^{\ell-1}\left(x_{\Gamma}\right) \text { and } \lim _{\eta \rightarrow \infty} w_{i}^{\ell}\left(x_{\Gamma}, \eta\right)=0
\end{array}\right.
$$

(here we use the convention $w_{i}^{\ell} \equiv 0$ for $\ell<0$ ) and let $w_{e}^{\ell} \in H_{\mathrm{loc}}^{1}\left(\mathbb{R}^{d} \backslash \Omega\right.$ ) be the unique outgoing solution to

$$
\begin{cases}\Delta w_{e}^{\ell}+k^{2} w_{e}^{\ell}=0 & \text { for } x \in \mathbb{R}^{d} \backslash \bar{\Omega} \\ w_{e}^{\ell}(x)=w_{i}^{\ell}(x, 0) & \text { for } x \in \Gamma .\end{cases}
$$

From (3.7), one has (see (4.27) and (4.28) in Ref. 12):

$$
w_{i}^{1}\left(x_{\Gamma}, \eta\right)=-\frac{1}{\alpha} \partial_{n} w_{e}^{0} e^{-\alpha \eta}
$$

and

$$
w_{i}^{2}\left(x_{\Gamma}, \eta\right)=\left\{-\frac{1}{\alpha} \partial_{n} w_{e}^{1}+\frac{\mathcal{H}}{\alpha^{2}} \partial_{n} w_{e}^{0}+\frac{\eta \mathcal{H}}{\alpha} \partial_{n} w_{e}^{0}\right\} e^{-\alpha \eta} .
$$

Let $\chi \in C_{0}^{\infty}(\mathbb{R})$ satisfy

$$
\chi(\eta)= \begin{cases}1 & \text { if }|\eta| \leq \delta / 2 \\ 0 & \text { if }|\eta| \geq \delta .\end{cases}
$$

Following Ref. 12, we set:

$$
\begin{aligned}
v_{\ell}^{e}(x) & =w_{e}^{0}(x)+\hat{\epsilon} w_{e}^{1}+\cdots+\hat{\epsilon}^{\ell} w_{e}^{\ell}(x), & & x \in \mathbb{R}^{d} \backslash \bar{\Omega}, \\
v_{\ell}^{i}(x) & =\left[w_{i}^{0}\left(x_{\Gamma}, \nu / \hat{\epsilon}\right)+\hat{\epsilon} w_{i}^{1}\left(x_{\Gamma}, \nu / \hat{\epsilon}\right)+\cdots+\hat{\epsilon}^{\ell} w_{i}^{\ell}\left(x_{\Gamma}, \nu / \hat{\epsilon}\right)\right] \chi(x), & & x \in \Omega .
\end{aligned}
$$

For $x \in \Omega^{\delta}$, define

$$
\varphi_{\hat{\epsilon}}(x)=\nu \chi(\nu / \hat{\epsilon}) \partial_{n} w_{e}^{\ell}\left(x_{\Gamma}\right) \quad \text { where } x=x_{\Gamma}+\nu n .
$$

It is clear that $\varphi_{\hat{\epsilon}} \in C^{\infty}(\bar{\Omega})$,

$$
\varphi_{\hat{\epsilon}}(x)=0 \quad \text { on } \Gamma \quad \text { and } \quad \partial_{n} \varphi_{\hat{\epsilon}}(x)=\partial_{n} w_{e}^{\ell} \quad \text { on } \Gamma .
$$


We define ${ }^{\mathrm{k}}$ :

$$
\mathbf{d}_{\ell}(x):= \begin{cases}v^{\epsilon}(x)-v_{e}^{\ell}(x) & \text { in } \mathbb{R}^{d} \backslash \Omega \\ v^{\epsilon}(x)-v_{i}^{\ell}(x)-\hat{\epsilon}^{\ell} \varphi_{\hat{\epsilon}}(x) & \text { in } \Omega\end{cases}
$$

and

$$
q_{\ell}:=\Delta \mathbf{d}_{\ell}+k^{2} \mathbf{d}_{\ell}+\frac{i}{\hat{\epsilon}^{2}} \chi_{\Omega} \mathbf{d}_{\ell} \quad \text { in } \mathbb{R}^{d}
$$

We also set

$$
\mathbf{e}_{\ell}:=v_{\ell}^{e}-v_{\ell}^{a} \quad \text { in } \mathbb{R}^{d} \backslash \Omega \quad \text { and } \quad h_{\ell}:=\mathbf{e}_{\ell}+\mathcal{D}_{\ell}^{\hat{\epsilon}} \partial_{n} \mathbf{e}_{\ell} \quad \text { on } \Gamma .
$$

Then $\mathbf{e}_{\ell} \in H_{\text {loc }}^{1}\left(\mathbb{R}^{d}\right), \mathbf{e}_{\ell}$ satisfies the outgoing condition, and

$$
\begin{cases}\Delta \mathbf{e}_{\ell}+k^{2} \mathbf{e}_{\ell}=0, & \text { in } \mathbb{R}^{d} \backslash \bar{\Omega}, \\ \mathbf{e}_{\ell}+\mathcal{D}_{\ell}^{\hat{\epsilon}} \partial_{n} \mathbf{e}_{\ell}=h_{\ell}, & \text { on } \Gamma .\end{cases}
$$

We now state estimates used later. We begin with two estimates on $w_{e}^{\ell}$. The first one, whose proof is given in Sec. A.1, deals with the low and moderate frequency regimes.

Lemma 3.1. Let $d=2,3, \ell=0,1,2, m \geq 0, k_{0}>0$, and $R>0$. There exist two positive constants $C_{R, \ell}$ and $C_{\ell, m}$ independent of $\epsilon$ and $k$ such that, for $0<k \leq k_{0}$,

$$
\left\|w_{e}^{\ell}\right\|_{H^{m+1}\left(B_{R} \backslash \Omega\right)} \leq C_{R, \ell, m}\|\mathbf{s}\|_{H^{2 \ell+m}\left(\mathbb{R}^{d}\right)}
$$

and, for $m \geq 1$,

$$
\left\|\partial_{n} w_{e}^{\ell}\right\|_{H^{m-1 / 2}(\Gamma)} \leq C_{\ell, m}\|\mathbf{s}\|_{H^{2 \ell+m}\left(\mathbb{R}^{d}\right)} .
$$

Here is the second estimate of $w_{e}^{\ell}$ in the high frequency regime whose proof is given in Sec. A.2.

Lemma 3.2. Let $d=2,3, \ell=0,1,2, m \geq 0, k_{0}>0$, and $R>0$. Assume that $\Omega$ is star-shaped. There exist two positive constants $C_{R, \ell}$ and $C_{\ell, m}$ independent of $\epsilon$ and $k$ such that, for $k \geq k_{0}$,

$$
\left\|w_{e}^{\ell}\right\|_{H^{m+1}\left(B_{R} \backslash \Omega\right)}+k\left\|w_{e}^{\ell}\right\|_{H^{m}\left(B_{R} \backslash \Omega\right)} \leq C_{R, \ell, m} k^{2 \ell+m}\|\mathbf{s}\|_{H^{2 \ell+m}\left(\mathbb{R}^{d}\right)}
$$

and, for $m \geq 1$,

$$
\left\|\partial_{n} w_{e}^{\ell}\right\|_{H^{m-1 / 2}(\Gamma)} \leq C_{\ell, m} k^{2 \ell+m}\|\mathbf{s}\|_{H^{2 \ell+m}\left(\mathbb{R}^{d}\right)}
$$

\footnotetext{
${ }^{\mathrm{k}}$ Our definition of $\mathbf{d}_{\ell}$ is slightly different from the one in Ref. 12 in $\Omega$. We include the term $-\hat{\epsilon}^{\ell} \varphi_{\hat{\epsilon}}(x)$ to make $\partial_{n} \mathbf{d}_{\ell}$ continuous across $\Gamma$ while maintaining the continuity of $\mathbf{d}_{\ell}$. This modification is convenient for the use of Morawetz's technique in the high frequency regime later. The scaling for the variable of function $\chi$ in the definition of $\varphi$ reflects the skin effect.
} 
The following two lemmas give us the essential estimates for $q_{\ell}$ and $h_{\ell}$. Their proofs are given in Secs. A.3 and A.4, respectively.

Lemma 3.3. We have $\operatorname{supp} q_{\ell} \subset \bar{\Omega}$. Moreover, let $0<\epsilon<1, k \geq 0, k_{0}>0, \ell=$ $0,1,2$. Assume that $m \geq 1$ and let $s \in H^{\ell+m}\left(\mathbb{R}^{d}\right)$. Then, there is a positive constant $C$ independent of $\epsilon$ and $k$, such that:

(i) for $\epsilon^{2}<k \leq k_{0}$, we have

$$
\left\|q_{\ell}\right\|_{L^{2}\left(\mathbb{R}^{d}\right)} \leq C \hat{\epsilon}^{\ell-1}\|\mathbf{s}\|_{H^{2 \ell+3}\left(\mathbb{R}^{d}\right)},
$$

(ii) assuming in addition that $\Omega$ is star-shaped, for $k \geq k_{0}$, we have

$$
\left\|q_{\ell}\right\|_{L^{2}\left(\mathbb{R}^{d}\right)} \leq C k^{2 \ell+3} \hat{\epsilon}^{\ell-1}\|\mathbf{s}\|_{H^{2 \ell+3}\left(\mathbb{R}^{d}\right)} .
$$

Lemma 3.4. Let $0<\epsilon<1, k \geq 0, k_{0}>0, \ell=0,1,2$, and $m \geq 1$. There is a positive constant $C_{\ell, m}$ independent of $\epsilon$ and $k$ such that:

(i) for $\epsilon^{2}<k \leq k_{0}$, we have

$$
\left\|h_{\ell}\right\|_{H^{m-1 / 2}(\Gamma)} \leq C_{\ell, m} \hat{\epsilon}^{\ell+1}\|\mathbf{s}\|_{H^{2 \ell+m}\left(\mathbb{R}^{d}\right)},
$$

(ii) assuming in addition that $\Omega$ is star-shaped, for $k \geq k_{0}$, we have

$$
\left\|h_{\ell}\right\|_{H^{m-1 / 2}(\Gamma)} \leq C_{\ell, m} k^{2 \ell+m} \hat{\epsilon}^{\ell+1}\|\mathbf{s}\|_{H^{2 \ell+m}\left(\mathbb{R}^{d}\right)} .
$$

\section{Proofs of Propositions 1.1 and 1.2}

This section containing three subsections is devoted to Propositions 1.1 and 1.2. In the first subsection, we present several useful lemmas. The proofs of Propositions 1.1 and 1.2 are given in the last two subsections.

\subsection{Preliminaries}

In this section, we present useful lemmas used in the proof of Propositions 1.1 and 1.2. We first recall the following results established in Ref. 21 (Lemma 2.2), see also Ref. 20 (Lemma 2.2).

Lemma 4.1. Let $d=2,3,0<k<k_{0}$, and $\Omega$ be a smooth bounded connected subset of $\mathbb{R}^{d}$. Let $g_{k} \in H^{\frac{1}{2}}(\partial \Omega)$ and $v_{k} \in H_{\text {loc }}^{1}\left(\mathbb{R}^{d} \backslash \Omega\right)$ be the unique outgoing solution to

$$
\begin{cases}\Delta v_{k}+k^{2} v_{k}=0 & \text { in } \mathbb{R}^{d} \backslash \bar{\Omega}, \\ v_{k}=g_{k} & \text { on } \Gamma .\end{cases}
$$

Assume that $g_{k} \rightarrow g$ weakly in $H^{\frac{1}{2}}(\partial \Omega)$ as $k \rightarrow 0$. Then $v_{k} \rightarrow v$ weakly in $H_{\text {loc }}^{1}\left(\mathbb{R}^{d} \backslash \Omega\right)$ where $v \in W^{1}\left(\mathbb{R}^{d} \backslash \bar{\Omega}\right)$ is the unique solution to

$$
\begin{cases}\Delta v=0 & \text { in } \mathbb{R}^{d} \backslash \bar{\Omega}, \\ v=g & \text { on } \Gamma .\end{cases}
$$


Here, for an open unbounded subset $U$ of $\mathbb{R}^{d}$, the space $W^{1}(U)$ is defined as follows: $W^{1}(U)$

$$
= \begin{cases}\left\{\psi \in L_{\mathrm{loc}}^{1}(U) ; \frac{\psi(x)}{\ln (2+|x|) \sqrt{1+|x|^{2}}} \in L^{2}(U) \text { and } \nabla \psi \in L^{2}(U)\right\} & \text { if } d=2, \\ \left\{\psi \in L_{\mathrm{loc}}^{1}(U) ; \frac{\psi(x)}{\sqrt{1+|x|^{2}}} \in L^{2}(U) \text { and } \nabla \psi \in L^{2}(U)\right\} & \text { if } d=3 .\end{cases}
$$

Using Lemma 4.1, we can prove the following.

Lemma 4.2. Let $d=2,3, \ell=0,1,0<\epsilon<1, k_{0}>0, r_{0}>0,0<k<k_{0}, q \in$ $L^{2}\left(\mathbb{R}^{d} \backslash \Omega\right)$ with $\operatorname{supp} q \subset B_{r_{0}} \backslash \Omega$, and $g \in H^{1 / 2}(\Gamma)$. Let $v \in H_{\mathrm{loc}}^{1}\left(\mathbb{R}^{d} \backslash \Omega\right)$ be the unique outgoing solution to

$$
\begin{cases}\Delta v+k^{2} v=q & \text { in } \mathbb{R}^{d} \backslash \Omega, \\ v+\mathcal{D}_{\ell}^{\hat{\epsilon}} \partial_{n} v=g & \text { on } \Gamma .\end{cases}
$$

We have

$$
\|v\|_{H^{1}\left(B_{r} \backslash \Omega\right)} \leq C_{r}\left(\max \{1, \hat{\epsilon}\}\|q\|_{L^{2}\left(\mathbb{R}^{d} \backslash \Omega\right)}+\|g\|_{H^{1 / 2}(\Gamma)}\right),
$$

for some positive constant $C_{r}$ independent of $g, q, \epsilon$ and $k$.

We recall here that, by $(1.6), \hat{\epsilon}=\epsilon / \sqrt{k}$.

Proof. Set $r_{1}:=r_{0}+1$. We first prove that

$$
\|v\|_{L^{2}\left(B_{r_{1}} \backslash \Omega\right)} \leq C\left(\max \{1, \hat{\epsilon}\}\|q\|_{L^{2}\left(\mathbb{R}^{d} \backslash \Omega\right)}+\|g\|_{H^{1 / 2}(\Gamma)}\right),
$$

for some positive constant $C$, independent of $\epsilon, k, q$ and $g$, by contradiction. Suppose this is not true. Then there exist $\epsilon_{n} \rightarrow \epsilon_{*} \in[0,1], k_{n} \rightarrow k_{*} \in\left[0, k_{0}\right], q_{n} \in L^{2}\left(\mathbb{R}^{d}\right)$ with $\operatorname{supp} q_{n} \subset B_{r_{0}} \backslash \Omega$, and $g_{n} \in H^{1 / 2}(\Gamma)$ such that

$$
\max \left\{1, \hat{\epsilon}_{n}\right\}\left\|q_{n}\right\|_{L^{2}\left(\mathbb{R}^{d} \backslash \Omega\right)}+\left\|g_{n}\right\|_{H^{1 / 2}(\Gamma)} \rightarrow 0 \quad \text { and } \quad\left\|v_{n}\right\|_{L^{2}\left(B_{r_{1}} \backslash \Omega\right)}=1 .
$$

Here, $v_{n} \in H_{\text {loc }}^{1}\left(\mathbb{R}^{d} \backslash \Omega\right)$ is the unique outgoing solution to the problem

$$
\begin{cases}\Delta v_{n}+k_{n}^{2} v_{n}=q_{n} & \text { in } \mathbb{R}^{d} \backslash \bar{\Omega}, \\ v_{n}+\mathcal{D}_{\ell}^{\hat{\epsilon}_{n}} \partial_{n} v_{n}=g_{n} & \text { on } \Gamma .\end{cases}
$$

Using the standard regularity theory of elliptic equations and the representation formula for the equation $\Delta v_{n}+k_{n}^{2} v_{n}=0$ in $\mathbb{R}^{d} \backslash B_{r_{0}}$, we have

$$
\left\|v_{n}\right\|_{H^{1}\left(B_{r} \backslash B_{r_{0}+1 / 2}\right)} \leq C_{r} \text { for all } r>0 .
$$


Multiplying the first equation of (4.6) by $\bar{v}_{n}$ (the conjugate of $v_{n}$ ), integrating over $B_{r_{1}} \backslash \Omega$, and using the second equation of (4.6), we obtain

$$
\begin{aligned}
\int_{B_{r_{1} \backslash \Omega}} \nabla v_{n} \nabla \bar{v}_{n}+\int_{\Gamma} \partial_{n} v_{n} \overline{\mathcal{D}_{\ell}^{\hat{\epsilon}_{n}} \partial_{n} v_{n}} & =\int_{\partial B_{r_{1}}} \partial_{n} v_{n} \bar{v}_{n}+k_{n}^{2} \int_{B_{r_{1}} \backslash \Omega}\left|v_{n}\right|^{2} \\
& -\int_{B_{r_{1} \backslash \Omega}} q_{n} \bar{v}_{n}+\int_{\Gamma} \partial_{n} v_{n} \overline{g_{n}}
\end{aligned}
$$

We have, by (1.6) and (1.7),

$$
\begin{aligned}
& \Re\left[\partial_{n} v_{n} \overline{\mathcal{D}_{\ell}^{\hat{\epsilon}_{n}} \partial_{n} v_{n}}\right]=\frac{\sqrt{2}}{2} \hat{\epsilon}_{n}\left|\partial_{n} v_{n}\right|^{2} \geq 0 \quad \text { and } \\
& \Im\left[\partial_{n} v_{n} \overline{\mathcal{D}_{\ell}^{\hat{\epsilon}_{n}} \partial_{n} v_{n}}\right]=-\frac{\sqrt{2}}{2} \hat{\epsilon}_{n}\left|\partial_{n} v_{n}\right|^{2} .
\end{aligned}
$$

Considering the real part of (4.8) and using (4.7) and (4.9) we have

$$
\int_{B_{r_{1} \backslash \Omega}}\left|\nabla v_{n}\right|^{2} \leq C .
$$

Here and what follows in the proof, $C$ denotes a positive constant independent of $n, \epsilon, k$.

Considering the imaginary part of (4.8), letting $r_{1} \rightarrow+\infty$, and using the outgoing condition, (4.7) and (4.9), we obtain

$$
\hat{\epsilon}_{n} \int_{\Gamma}\left|\partial_{n} v_{n}\right|^{2} \leq \sqrt{2} \int_{\Gamma}\left|\partial_{n} v_{n}\right|\left|g_{n}\right|+\left\|q_{n}\right\|_{L^{2}} .
$$

This implies

$$
\hat{\epsilon}_{n}\left\|\partial_{n} v_{n}\right\|_{L^{2}(\Gamma)} \leq C\left(\left\|g_{n}\right\|_{L^{2}(\Gamma)}+\hat{\epsilon}_{n}^{1 / 2}\left\|q_{n}\right\|_{L^{2}}^{1 / 2}\right) \rightarrow 0 \quad \text { by }(4.5) .
$$

We now divide the proof into two cases: (1) $k_{*}=0$, and $(2) k_{*}>0$.

Let first consider case (1). From (4.7) and (4.10), without loss of generality one may assume that $v_{n} \rightarrow v$ weakly in $H_{\text {loc }}^{1}\left(\mathbb{R}^{d} \backslash \Omega\right)$. From (4.5), (4.6) and (4.11), one obtains

$$
\begin{cases}\Delta v=0 & \text { in } \mathbb{R}^{d} \backslash \bar{\Omega} \\ v=0 & \text { on } \Gamma\end{cases}
$$

Moreover, applying Lemma 4.1,

$$
v \in W^{1}\left(\mathbb{R}^{d} \backslash \bar{\Omega}\right) .
$$

This implies

$$
v=0 \text { in } \mathbb{R}^{d} \backslash \Omega .
$$

On the other hand, we derive from (4.5) that

$$
\|v\|_{L^{2}\left(B_{r_{1}} \backslash \Omega\right)}=\lim _{n \rightarrow \infty}\left\|v_{n}\right\|_{L^{2}\left(B_{\left.r_{1} \backslash \Omega\right)}\right.}=1 .
$$

We have a contradiction. Thus (4.4) holds. 
Considering case (2), we obtain, instead of (4.12),

$$
\begin{cases}\Delta v+k_{*}^{2} v=0 & \text { in } \mathbb{R}^{d} \backslash \bar{\Omega}, \\ v=0 & \text { on } \Gamma,\end{cases}
$$

and $v$ satisfies the outgoing condition. Hence $v=0$. We also have a contradiction as in case (1). Therefore, (4.4) holds.

From (4.4), as in the proof of (4.7) and (4.10), we obtain (4.3). The proof is complete.

We now state the last results in this section dealing with (1.9) in the low and moderate frequency regimes.

Lemma 4.3. Let $d=2,3,0<\epsilon<1, k_{0}>0, r_{0}>0,0<k<k_{0}$, and let $q \in L^{2}\left(\mathbb{R}^{d}\right)$ with $\operatorname{supp} q \subset B_{r_{0}}$. Let $v \in H_{\text {loc }}^{1}\left(\mathbb{R}^{d} \backslash \Omega\right)$ be the unique outgoing solution to

$$
\Delta v+k^{2} v+i k \sigma_{\epsilon} v=q \quad \text { in } \mathbb{R}^{d} .
$$

We have, for $r>0$,

$$
\|v\|_{H^{1}\left(B_{r}\right)} \leq C_{r} \max \left\{1, \hat{\epsilon}^{2}\right\}\left(\|q\|_{L^{2}\left(\Omega^{c}\right)}+\hat{\epsilon}\|q\|_{L^{2}(\Omega)}\right),
$$

where $C_{r}$ is a positive constant independent of $\epsilon, k$ and $q$.

Proof. The proof of Lemma 4.3 is similar to one of Lemma 4.2. The details are left to the reader.

\subsection{Proof of Proposition 1.1}

Proposition 1.1 is a direct consequence of Lemmas 4.2 and 4.3. Indeed, applying Lemma 4.2 for $v=v_{\ell}^{a}, q=\mathbf{s}$, we obtain, for $\ell=0,1$,

$$
\left\|v_{\ell}^{a}\right\|_{H^{1}\left(B_{r} \backslash \Omega\right)} \leq C \hat{\epsilon}\|\mathbf{s}\|_{L^{2}\left(\mathbb{R}^{d}\right)} \leq C \hat{\epsilon}^{2}\|\mathbf{s}\|_{L^{2}\left(\mathbb{R}^{d}\right)},
$$

and, applying Lemma 4.3 for $v=v^{\epsilon}$ and $q=\mathbf{s}$, we have

$$
\left\|v^{\epsilon}\right\|_{H^{1}\left(B_{r}\right)} \leq C \hat{\epsilon}^{2}\|\mathbf{s}\|_{L^{2}\left(\mathbb{R}^{d}\right)} .
$$

A combination of (4.15) and (4.16) yields the conclusion.

\subsection{Proof of Proposition 1.2}

It is from the definition of $\mathbf{d}_{\ell}(3.14)$ and $\mathbf{e}_{\ell}$ (3.16) that

$$
\left\|v^{\epsilon}-v_{\ell}^{a}\right\|_{H^{1}\left(B_{r} \backslash \Omega\right)} \leq\left\|\mathbf{d}_{\ell}\right\|_{H^{1}\left(B_{r} \backslash \Omega\right)}+\left\|\mathbf{e}_{\ell}\right\|_{H^{1}\left(B_{r} \backslash \Omega\right)} .
$$

Applying Lemmas 4.2 and 3.4 (with $m=1$ ), from $(3.17$ ), we have for $\ell=0,1$,

$$
\left\|\mathbf{e}_{\ell}\right\|_{H^{1}\left(B_{r} \backslash \Omega\right)} \leq C\left\|h_{\ell}\right\|_{H^{1 / 2}(\Gamma)} \leq C \hat{\epsilon}^{\ell+1}\|\mathbf{s}\|_{H^{2 \ell+1}\left(\mathbb{R}^{d}\right)} .
$$


Using Lemmas 4.3 and 3.3, from (3.15), we obtain, for $\ell=0,1,2$,

$$
\left\|\mathbf{d}_{\ell}\right\|_{H^{1}\left(B_{r} \backslash \Omega\right)} \leq C \hat{\epsilon}\left\|q_{\ell}\right\|_{L^{2}(\Omega)} \leq C \hat{\epsilon}^{\ell}\|\mathbf{s}\|_{H^{2 \ell+3}\left(\mathbb{R}^{d}\right)} .
$$

By Lemma 3.1, it follows that, for $\ell=0,1$,

$$
\begin{aligned}
\left\|\mathbf{d}_{\ell}\right\|_{H^{1}\left(B_{r} \backslash \Omega\right)} & \leq \hat{\epsilon}^{\ell+1}\left\|w_{e}^{\ell+1}\right\|_{H^{1}\left(B_{r} \backslash \Omega\right)}+C \hat{\epsilon}^{\ell+1}\|\mathbf{s}\|_{H^{2 \ell+5}\left(\mathbb{R}^{d}\right)} \\
& \leq C \hat{\epsilon}^{\ell+1}\|\mathbf{s}\|_{H^{2 \ell+5}\left(\mathbb{R}^{d}\right)} .
\end{aligned}
$$

A combination of (4.17), (4.18) and (4.19) yields the conclusion.

\section{Proof of Proposition 1.3}

This section is devoted to the proof of Proposition 1.3. In order to obtain the desired estimate for $u_{a}^{\ell}-u^{\epsilon}$, we will derive separate estimates for the functions $\mathbf{d}_{\ell}$ and $\mathbf{e}_{\ell}$, introduced in Sec. 3. This goal is achieved by Corollaries 5.1 and 5.2 below. Our presentation is divided into two subsections. In the first one, we present some useful lemmas. The proof of Proposition 1.3 is given in the second subsection.

\subsection{Preliminaries}

In this section, we present useful lemmas used in the proof of Proposition 1.3. We start this section with the following lemma.

Lemma 5.1. Let $d=2,3, D$ be a star-shaped domain of $\mathbb{R}^{d}$, and $r_{*}>0$ such that $D \subset B_{r_{*}}$. Define

$$
P(r)=\left\{\begin{array}{ll}
\frac{2 r_{*}}{d-1} & \text { if } r>r_{*}, \\
\frac{2 r}{d-1} & \text { if } 0<r<r_{*},
\end{array} \quad \text { and } \quad Q(r)= \begin{cases}\frac{r_{*}}{r} & \text { if } r>r_{*}, \\
1 & \text { if } 0<r<r_{*},\end{cases}\right.
$$

and let $v \in H_{\mathrm{loc}}^{1}\left(\mathbb{R}^{d}\right)$ be such that $\Delta v+k^{2} v \in L_{\mathrm{loc}}^{2}\left(\mathbb{R}^{d}\right)$. For any $R>r_{*}$ and $k>0$, we have

$$
\begin{aligned}
& \Re \int_{B_{R} \backslash \Omega}\left(\Delta v+k^{2} v\right)\left[P(r) \bar{v}_{r}+Q(r) \bar{v}\right] \\
& \leq-\frac{1}{d-1} \int_{B_{r_{*} \backslash \Omega}}\left(|\nabla v|^{2}+k^{2}|v|^{2}\right) \\
& \quad+\frac{r_{*}(3-d)}{2} \int_{B_{R} \backslash B_{r_{*}}} \frac{u^{2}}{r^{3}}+F_{0}(v)-F(R, v) .
\end{aligned}
$$

Here, $n$ denotes the inward unit normal vector of $\partial D$,

$F_{0}(v)=\Re\left(\int_{\partial D} \frac{2}{d-1} \partial_{n} v(x \cdot \nabla \bar{v})-\frac{1}{d-1}(x \cdot n)|\nabla v|^{2}+\partial_{n} v \bar{v}+\frac{k^{2}}{d-1}(x \cdot n)|v|^{2}\right)$

and

$F(r, v)=\Re\left(-\int_{\partial B_{r}} \frac{r_{*}}{d-1}\left|v_{r}\right|^{2}-\frac{r_{*}}{d-1}\left|\nabla_{\partial B_{r}} v\right|^{2}+\frac{r_{*}}{2 r^{2}}|v|^{2}+\frac{r_{*}}{r} v_{r} \bar{v}+\frac{k^{2} r_{*}}{d-1}|v|^{2}\right)$. 
Remark 5.1. This lemma has been stated and proved in Ref. 22 (Lemma 1) for the spherical domains. The proof presented here follows heavily from the one of Ref. 22 (Lemma 1). In the proof, we also use the "Rellich" identity (5.2) which has root from Refs. 28, 26, and 19. Estimate (5.1) is in the spirit of Morawetz-Ludwig. ${ }^{19}$ The choice of the weight functions $P(r), Q(r)$ appeared in the work of Perthame and Vega. ${ }^{27}$

Proof of Lemma 5.1. We will prove the lemma for $v \in C^{\infty}\left(\mathbb{R}^{d}\right)$. The general case follows by a standard regularizing argument. We have

$$
\Re \int_{B_{R} \backslash D}\left(\Delta v+k^{2} v\right)\left[P(r) \bar{v}_{r}+Q(r) \bar{v}\right]=A_{1}+A_{2},
$$

where

$$
A_{1}=\Re \int_{B_{r_{*} \backslash D}}\left(\Delta v+k^{2} v\right)\left[P(r) \bar{v}_{r}+Q(r) \bar{v}\right]
$$

and

$$
A_{2}=\Re \int_{B_{R} \backslash B_{r_{*}}}\left(\Delta v+k^{2} v\right)\left[P(r) \bar{v}_{r}+Q(r) \bar{v}\right] .
$$

Calculate $\mathbf{A}_{\mathbf{1}}$ : Since $P(r)=\frac{2 r}{d-1}$ and $Q(r)=1$ for $0<r<r_{*}$,

$$
\begin{aligned}
A_{1} & =\Re \int_{B_{r_{*}} \backslash D}\left(\Delta v+k^{2} v\right)\left[\frac{2 r}{d-1} \bar{v}_{r}+\bar{v}\right] \\
& =\int_{B_{r_{*}} \backslash D} \Re\left[\left(\Delta v+k^{2} v\right)\left(\frac{2}{d-1} x \cdot \nabla \bar{v}+\bar{v}\right)\right] .
\end{aligned}
$$

We have ${ }^{\mathrm{l}}$ :

$$
\begin{aligned}
\Re\left[\left(\Delta v+k^{2} v\right)\left(\frac{2}{d-1} x \cdot \nabla \bar{v}+\bar{v}\right)\right] \\
=-\frac{1}{d-1}\left(|\nabla v|^{2}+k^{2}|v|^{2}\right)+\Re \nabla \\
\quad \times\left[\frac{2}{d-1} \nabla v(x \cdot \nabla \bar{v})-\frac{1}{d-1} x|\nabla v|^{2}+\nabla v \bar{v}+\frac{k^{2}}{d-1} x|v|^{2}\right] .
\end{aligned}
$$

Integrating over the domain $B_{r_{*}} \backslash D$, we obtain

$$
\begin{aligned}
A_{1}= & -\frac{1}{d-1} \int_{B_{r_{*}} D}\left(|\nabla v|^{2}+k^{2}|v|^{2}\right)+\Re \int_{\partial B_{r_{*}}} \frac{2 r_{*}}{d-1}\left|v_{r}\right|^{2}-\frac{r_{*}}{d-1}|\nabla v|^{2}+v_{r} \bar{v} \\
& +\frac{k^{2} r_{*}}{d-1}|v|^{2}+\Re \int_{\partial D} \frac{2}{d-1} \partial_{n} v(x \cdot \nabla \bar{v})-\frac{1}{d-1}(x \cdot n)|\nabla v|^{2} \\
& +\partial_{n} v \bar{v}+\frac{k^{2}}{d-1}(x \cdot n)|v|^{2} .
\end{aligned}
$$

${ }^{1}$ This is the "Rellich" identity which has root from Refs. 28, 26, and 19. 
It follows that

$$
\begin{aligned}
A_{1}= & -\frac{1}{d-1} \int_{B_{r_{*}} \backslash D}\left(|\nabla v|^{2}+k^{2}|v|^{2}\right)+F_{0}(v) \\
& +\Re \int_{\partial B_{r_{*}}} \frac{2 r_{*}}{d-1}\left|v_{r}\right|^{2}-\frac{r_{*}}{d-1}|\nabla v|^{2}+v_{r} \bar{v}+\frac{k^{2} r_{*}}{d-1}|v|^{2} .
\end{aligned}
$$

Since $|\nabla v|^{2}=\left|v_{r}\right|^{2}+\left|\nabla_{\partial B_{r_{*}}} v\right|^{2}$, we have

$$
\begin{aligned}
& \Re \int_{\partial B_{r_{*}}} \frac{2 r_{*}}{d-1}\left|v_{r}\right|^{2}-\frac{r_{*}}{d-1}|\nabla v|^{2}+v_{r} \bar{v}+\frac{k^{2} r_{*}}{d-1}|v|^{2} \\
& \quad=\Re \int_{\partial B_{r_{*}}} \frac{r_{*}}{d-1}\left|v_{r}\right|^{2}-\frac{r_{*}}{d-1}\left|\nabla_{\partial B_{r_{*}}} v\right|^{2}+v_{r} \bar{v}+\frac{r_{*} k^{2}}{d-1}|v|^{2} \leq-F\left(r_{*}, v\right) .
\end{aligned}
$$

It follows that

$$
A_{1} \leq-\frac{1}{d-1} \int_{B_{r_{*} \backslash D}}\left(|\nabla v|^{2}+k^{2}|v|^{2}\right)+F_{0}(v)-F\left(r_{*}, v\right) .
$$

Estimate $\mathbf{A}_{\mathbf{2}}$ : Applying Lemma 2 in Ref. 22, we have

$$
A_{2} \leq \frac{r_{*}(3-d)}{2} \int_{B_{R} \backslash B_{r_{*}}} \frac{u^{2}}{r^{3}}+F\left(r_{*}, v\right)-F(R, v) .
$$

The conclusion now follows from (5.3) and (5.4).

The following lemma, in spirit of Morawetz-Ludwig ${ }^{19}$ (see also Ref. 27), is important for our analysis.

Lemma 5.2. Let $d=2,3, k_{0}>0, r_{0}>0, q \in L^{2}\left(\mathbb{R}^{d}\right)$ with $\operatorname{supp} q \subset B_{r_{0}} \backslash \Omega$, and $g \in H^{1}(\Gamma)$. Let $k \geq k_{0}$ and $v \in H_{\mathrm{loc}}^{1}\left(\mathbb{R}^{d} \backslash \Omega\right)$ be the unique outgoing solution to

$$
\begin{cases}\Delta v+k^{2} v=q & \text { in } \mathbb{R}^{d} \backslash \bar{\Omega} \\ v=g & \text { on } \Gamma\end{cases}
$$

Assume that $\Omega$ is star-shaped. Given $r_{*}>0$, there exists a positive constant $C=$ $C\left(r_{*}, r_{0}, k_{0}, \Omega\right)$ independent of $k$ such that

$$
\begin{aligned}
& \|\nabla v\|_{L^{2}\left(B_{r_{*}} \backslash \Omega\right)}+k\|v\|_{L^{2}\left(B_{r_{*}} \backslash \Omega\right)}+\left\|\partial_{n} v\right\|_{L^{2}(\Gamma)} \\
& \quad \leq C\left(\|q\|_{L^{2}\left(\mathbb{R}^{d} \backslash \Omega\right)}+\left\|\nabla_{\Gamma} g\right\|_{L^{2}(\Gamma)}+k\|g\|_{L^{2}(\Gamma)}\right) .
\end{aligned}
$$

Proof. The idea is to apply Lemma 5.1 for $D=\Omega$. We have

$$
\begin{aligned}
F_{0}(v)= & \Re \int_{\partial \Omega}\left(\frac{1}{d-1}(x \cdot n)\left|\partial_{n} v\right|^{2}+\frac{2}{d-1} \partial_{n} v \nabla_{\Gamma} \bar{v}-\frac{1}{d-1}(x \cdot n)\left|\nabla_{\Gamma} v\right|^{2}\right. \\
& \left.+\partial_{n} v \bar{v}+\frac{k^{2}}{d-1}(x \cdot n)|v|^{2}\right),
\end{aligned}
$$


where $n$ denotes the inward unit normal vector of $\partial \Omega$. Since $\Omega$ is star-shaped, it follows that

$$
F_{0}(v) \leq-\frac{1}{C}\left\|\partial_{n} v\right\|^{2}+C\left(\left\|\nabla_{\Gamma} v\right\|_{L^{2}(\Gamma)}+k^{2}\|v\|_{L^{2}(\Gamma)}\right)
$$

Applying Lemma 5.1 and using (5.7), we obtain

$$
\begin{aligned}
& \|\nabla v\|_{L^{2}\left(B_{r_{*}} \backslash \Omega\right)}^{2}+k^{2}\|v\|_{L^{2}\left(B_{\left.r_{*} \backslash \Omega\right)}\right.}^{2}+\left\|\partial_{n} v\right\|_{L^{2}(\Gamma)}^{2} \\
& \quad \leq C\left(\frac{r_{*}(3-d)}{2} \int_{B_{R} \backslash B_{r_{*}}} \frac{u^{2}}{r^{3}}+\|q\|_{L^{2}\left(\mathbb{R}^{d} \backslash \Omega\right)}^{2}+\left\|\nabla_{\Gamma} g\right\|_{L^{2}(\Gamma)}^{2}+k\|g\|_{L^{2}(\Gamma)}^{2}\right) .
\end{aligned}
$$

This implies the conclusion in the case $d=3$. For $d=2$, it remains to absorb the first term in the right-hand side into the left-hand side. Without loss of generality, we may assume that $r_{*}$ is large enough. The absorption then can be done as in pp. 11-12 of Ref. 22. The details are left to the reader.

When the control is only available on $L^{2}(\Gamma)$ (not $H^{1}(\Gamma)$ ), one has the following result by Hörmander (see Theorem 3.1 and p. 65 in Ref. 15).

Lemma 5.3. (Hörmander) Let $D$ be a bounded smooth domain of $\mathbb{R}^{d}(d \geq 2)$ and $g \in H^{1 / 2}(\partial D)$. Assume $v \in H^{1}(D)$ is the unique solution to the system

$$
\begin{cases}\Delta v=0 & \text { in } D, \\ v=g & \text { on } \partial D .\end{cases}
$$

Then

$$
\|v\|_{L^{2}(D)} \leq C\|g\|_{L^{2}(\partial D)},
$$

for some positive constant $C$ independent of $g .^{\mathrm{m}}$

Here is a result related to Eq. (3.17) of $\mathbf{e}_{\ell}$.

Lemma 5.4. Let $d=2,3, \ell=0,1,0<\epsilon<1, k_{0}>0, k \geq k_{0}, h \in L^{2}(\Gamma)$, and let $v \in H_{\mathrm{loc}}^{1}\left(\mathbb{R}^{d} \backslash \Omega\right)$ be the unique outgoing solution to

$$
\begin{cases}\Delta v+k^{2} v=0 & \text { in } \mathbb{R}^{d} \backslash \bar{\Omega}, \\ v+\mathcal{D}_{\ell}^{\hat{\epsilon}} \partial_{n} v=h & \text { on } \Gamma .\end{cases}
$$

We have

$$
\|v\|_{L^{2}(\Gamma)} \leq C\|h\|_{L^{2}(\Gamma)}
$$

where $C$ is a positive constant, independent of $\epsilon, k, q$ and $h$.

${ }^{\mathrm{m}} \operatorname{In}(5.9),\|g\|_{L^{2}(\partial D)}$ is used, not $\|g\|_{H^{1 / 2}(\partial D)}$. 
Proof. We only consider the case $\ell=1$ since the lemma is trivial for $\ell=0$. Let $\ell=1$. Multiplying the first equation of (5.10) by $\bar{v}$, integrating in $B_{r} \backslash \Omega$, and using the boundary condition, we obtain

$$
\int_{B_{r} \backslash \bar{\Omega}}-|\nabla v|^{2}+k^{2}|v|^{2}+\int_{\partial B_{r}} \partial_{r} v \bar{v}+\int_{\Gamma} \partial_{n} v \bar{h}-\int_{\Gamma} \partial_{n} v \overline{\mathcal{D}_{1}^{\hat{\epsilon}} \partial_{n} v}=0 .
$$

Recall that

$$
\mathcal{D}_{1}^{\hat{\epsilon}}=\frac{\hat{\epsilon}}{\alpha}=\frac{\sqrt{2}}{2} \hat{\epsilon}+i \frac{\sqrt{2}}{2} \hat{\epsilon}
$$

which implies

$$
-\Im\left[\partial_{n} v \overline{\mathcal{D}_{\ell}^{\hat{\epsilon}} \partial_{n} v}\right]=\frac{\sqrt{2}}{2} \hat{\epsilon}\left|\partial_{n} v\right|^{2}
$$

Since $v$ satisfies the outgoing condition (1.5), i.e.

$$
\partial_{r} v-i k v=o\left(r^{-(d-1) / 2}\right)
$$

it follows that

$$
\liminf _{r \rightarrow \infty} \Im\left(\int_{\partial B_{r}} \partial_{r} v \bar{v}\right)=\liminf _{r \rightarrow \infty} \int_{\partial B_{r}} k|v|^{2} \geq 0 .
$$

Considering the imaginary part of (5.11) and letting $r \rightarrow \infty$, we derive from (5.13) and (5.14) that

$$
\int_{\Gamma} \hat{\epsilon}\left|\partial_{n} v\right|^{2} \leq C \int_{\Gamma}|h|\left|\partial_{n} v\right|
$$

This implies

$$
\hat{\epsilon}\left\|\partial_{n} v\right\|_{L^{2}(\Gamma)} \leq\|h\|_{L^{2}(\Gamma)} .
$$

Since $v=h-\mathcal{D}_{\ell}^{\hat{\epsilon}} \partial_{n} v$, the conclusion follows from (5.12) and (5.15).

Here is an important consequence of Lemmas $5.2-5.4$, which will be applied to obtain the estimate for $\mathbf{e}_{\ell}$.

Corollary 5.1. Let $\ell=0,1, k_{0}>0,0<\epsilon<1, k \geq k_{0}, h \in H^{1 / 2}(\Gamma)$, and let $v \in H_{\mathrm{loc}}^{1}\left(\mathbb{R}^{d} \backslash \Omega\right)$ be the unique outgoing solution to

$$
\begin{cases}\Delta v+k^{2} v=0 & \text { in } \mathbb{R}^{d} \backslash \bar{\Omega} \\ v+\mathcal{D}_{\ell}^{\hat{\epsilon}} \partial_{n} v=h & \text { on } \Gamma\end{cases}
$$

Assume that $\Omega$ is star-shaped. Then, for all $K \subset \subset \mathbb{R}^{d} \backslash \bar{\Omega}$,

$$
\|\nabla v\|_{L^{2}(K)}+k\|v\|_{L^{2}(K)} \leq C k^{2}\|h\|_{L^{2}(\Gamma)},
$$

for some positive constant $C=C(K)$ independent of $\epsilon, k$ and $h$. 
Proof. Let $r>0$ such that $K \subset B_{r} \backslash \Omega$. Set $r_{1}=r+1$ and let $\phi \in H^{1}\left(B_{r_{1}} \backslash \Omega\right)$ be the solution to

$$
\left\{\begin{array}{l}
\Delta \phi=0 \text { in } B_{r_{1}} \backslash \bar{\Omega} \\
\phi=v \quad \text { on } \Gamma \quad \text { and } \quad \phi=0 \quad \text { on } \partial B_{r_{1}} .
\end{array}\right.
$$

Applying Lemma 5.4 we have

$$
\|v\|_{L^{2}(\Gamma)} \leq C\|h\|_{L^{2}(\Gamma)}
$$

It follows from Lemma 5.3 that

$$
\|\phi\|_{L^{2}\left(B_{r_{1}} \backslash \Omega\right)} \leq C\|h\|_{L^{2}(\Gamma)} .
$$

Fix $\chi \in C^{\infty}\left(\mathbb{R}^{d}\right)$ such that $\chi=1$ in $B_{r}$ and supp $\chi \subset B_{r+1 / 2}$. Set

$$
V=v-\chi \phi \quad \text { in } \mathbb{R}^{d} \backslash \Omega \text {. }
$$

It is clear that $V \in H_{\text {loc }}^{1}\left(\mathbb{R}^{d} \backslash \Omega\right)$ is the unique outgoing solution to the problem

$$
\begin{cases}\Delta V+k^{2} V=-\Delta(\chi \phi)-k^{2} \chi \phi & \text { in } \mathbb{R}^{d} \backslash \bar{\Omega}, \\ V=0 & \text { on } \Gamma .\end{cases}
$$

Since $\Delta \phi=0$ in $B_{r_{1}} \backslash \Omega, \chi=1$ in $B_{r}$, and $\chi=0$ in $\mathbb{R}^{d} \backslash B_{r+1 / 2}$,

$$
\|\Delta(\chi \phi)\|_{L^{2}\left(\mathbb{R}^{d} \backslash \Omega\right)}+k^{2}\|\chi \phi\|_{L^{2}\left(\mathbb{R}^{d} \backslash \Omega\right)} \leq C\left(\|\phi\|_{H^{2}\left(B_{r+1 / 2} \backslash B_{r}\right)}+k^{2}\|\phi\|_{L^{2}\left(B_{r_{1}} \backslash \Omega\right)}\right) .
$$

Using the standard regularity of elliptic equations, we derive from (5.17) that

$$
\|\Delta(\chi \phi)\|_{L^{2}\left(\mathbb{R}^{d} \backslash \Omega\right)}+k^{2}\|\chi \phi\|_{L^{2}\left(\mathbb{R}^{d} \backslash \Omega\right)} \leq C k^{2}\|\phi\|_{L^{2}\left(B_{r_{1}} \backslash \Omega\right)} .
$$

Applying Lemma 5.2 for (5.20) and using (5.21), we arrive to:

$$
\|\nabla V\|_{L^{2}\left(B_{r} \backslash \Omega\right)}+k\|V\|_{L^{2}\left(B_{r} \backslash \Omega\right)} \leq C k^{2}\|h\|_{L^{2}(\Gamma)} .
$$

The conclusion now follows from $(5.17),(5.19),(5.22)$, and the standard regularity theory for elliptic equations.

The following lemma, which is a variant of Proposition 1 in Ref. 23, plays an important role in analyzing $\mathbf{d}_{\ell}$.

Lemma 5.5. Let $d=2,3, k_{0}>0, r_{0}>0,0<\epsilon<1, k \geq k_{0}$, and $q \in L^{2}\left(\mathbb{R}^{d}\right)$ with $\operatorname{supp} q \subset \Omega$. Let $v \in H_{\text {loc }}^{1}\left(\mathbb{R}^{d}\right)$ be the unique outgoing solution to

$$
\Delta v+k^{2} v+i k \sigma_{\epsilon} v=q \quad \text { in } \mathbb{R}^{d} .
$$

We have, for $r_{*}>0$,

$$
\|\nabla v\|_{L^{2}\left(B_{r_{*}}\right)}+k\|v\|_{L^{2}\left(B_{r_{*}}\right)} \leq C_{r_{*}}\|q\|_{L^{2}(\Omega)}
$$

and

$$
\|v\|_{L^{2}(\Omega)} \leq C \epsilon^{2}\|q\|_{L^{2}(\Omega)}
$$

where $C_{r_{*}}$ and $C$ are positive constants independent of $k, \epsilon$ and $q$. As a consequence,

$$
\|v\|_{L^{2}(\Gamma)} \leq C \epsilon\|q\|_{L^{2}(\Omega)} .
$$


Proof. We follow the strategy used in the proof of Proposition 1 in Ref. 23. Multiplying Eq. (5.23) by $\bar{v}$ and integrating over $B_{R}$, we have

$$
-\int_{B_{R}}|\nabla v|^{2}+k^{2} \int_{B_{R}}|v|^{2}+\int_{\partial B_{R}} \partial_{r} v \bar{v}+i k \int_{\Omega} \sigma_{\epsilon}|v|^{2}=\int_{\Omega} q \bar{v} .
$$

Letting $R \rightarrow \infty$, using the outgoing condition, and considering the imaginary part, we obtain

$$
k \limsup _{R \rightarrow \infty} \int_{\partial B_{R}}|v|^{2}+\frac{1}{\hat{\epsilon}^{2}} \int_{\Omega}|v|^{2} \leq\|v\|_{L^{2}(\Omega)}\|q\|_{L^{2}(\Omega)} .
$$

This implies

$$
\int_{\Omega}|v|^{2} \leq \hat{\epsilon}^{4} \int_{\Omega}|q|^{2} \quad \text { and } \quad k \limsup _{R \rightarrow \infty} \int_{\partial B_{R}}|v|^{2} \leq \hat{\epsilon}^{2} \int_{\Omega}|q|^{2} .
$$

Hence (5.25) is proved. It only remains to prove (5.24).

Let $\gamma_{0}>0$ such that $B_{2 \gamma_{0}} \subset \Omega$. Multiplying (5.23) by $\phi^{2} \bar{v}$, with $\phi \in C_{\mathrm{c}}^{\infty}\left(B_{2 \gamma_{0}}\right)$ and $\phi=1$ in $B_{\gamma_{0}}$, and integrating over $\Omega$, we obtain, by Caccioppoli's inequality,

$$
\int_{B_{\gamma_{0}}}|\nabla v|^{2} \leq C\left(k^{2} \int_{\Omega}|v|^{2}+\int_{\Omega}|q|^{2}\right) .
$$

In this proof, $C$ denotes a positive constant independent of $\epsilon, k$ and $q$. It follows from (5.28) that

$$
\int_{B_{\gamma_{0}}}|\nabla v|^{2}+k^{2} \int_{B_{\gamma_{0}}}|v|^{2} \leq C\left(\hat{\epsilon}^{4} k^{2}+1\right) \int_{\Omega}|q|^{2}=C\left(\epsilon^{4}+1\right) \int_{\Omega}|q|^{2} \leq C \int_{\Omega}|q|^{2} .
$$

Hence, there exists $\gamma_{0} / 2<\tau \leq \gamma_{0}$ such that

$$
\int_{\partial B_{\tau}}|\nabla v|^{2}+k^{2} \int_{\partial B_{\tau}}|v|^{2} \leq C \int_{\Omega}|q|^{2}
$$

Applying Lemma 5.1 with $D=B_{\tau}$, we obtain, for any $R>r_{*}>R_{0}$,

$$
\begin{aligned}
\Re \int_{B_{R} \backslash B_{\tau}}\left[r \bar{v}_{r}+\bar{v}\right]\left[\Delta v+k^{2} v\right] \leq & -\frac{1}{d-1} \int_{B_{r_{*} \backslash B_{\tau}}}\left(|\nabla v|^{2}+k^{2}|v|^{2}\right) \\
& +\frac{r(3-d)}{2} \int_{B_{R} \backslash B_{r_{*}}} \frac{u^{2}}{r^{3}}+F_{0}(v)-F(R, v) .
\end{aligned}
$$

Here $F_{0}(v)$ and $F(R, v)$ are defined in Lemma 5.1. Using (5.23), we derive from (5.30) that

$$
\begin{aligned}
\frac{1}{d-1} \int_{B_{r_{*} \backslash B_{\tau}}\left(|\nabla v|^{2}+k^{2}|v|^{2}\right) \leq} & -\Re \int_{\Omega \backslash B_{\tau}}\left[r \bar{v}_{r}+\bar{v}\right]\left[-\frac{i}{\hat{\epsilon}^{2}} v+q\right] \\
& +\frac{r_{*}(3-d)}{2} \int_{B_{R} \backslash B_{r_{*}}} \frac{u^{2}}{r^{3}}+F_{0}(v)-F(R, v) .
\end{aligned}
$$


We have

$$
\left|\Re \int_{\Omega \backslash B_{\tau}}\left[r \bar{v}_{r}+\bar{v}\right]\left[-\frac{i}{\hat{\epsilon}^{2}} v+q\right]\right| \leq \frac{C}{\hat{\epsilon}^{2}} \int_{\Omega \backslash B_{\tau}}\left|v_{r}\right||v|+C \int_{\Omega \backslash B_{\tau}}\left(\left|v_{r}\right|+|v|\right)|q| .
$$

We derive from Young's inequality that

$$
\begin{aligned}
& \left|\Re \int_{\Omega \backslash B_{\tau}}\left[r \bar{v}_{r}+\bar{v}\right]\left[-\frac{i}{\hat{\epsilon}^{2}} v+q\right]\right| \\
& \quad \leq \frac{1}{2(d-1)} \int_{\Omega \backslash B_{\tau}}\left|v_{r}\right|^{2}+C\left(\frac{1}{\hat{\epsilon}^{4}} \int_{\Omega \backslash B_{\tau}}|v|^{2}+\int_{\Omega \backslash B_{\tau}}|q|^{2}\right) .
\end{aligned}
$$

A combination of (5.28), (5.31) and (5.32) yields

$$
\begin{aligned}
& \frac{1}{2(d-1)} \int_{B_{r_{*}} \backslash B_{\tau}}\left(|\nabla v|^{2}+k^{2}|v|^{2}\right) \\
& \quad \leq C \int_{\Omega}|q|^{2}+\frac{r_{*}(3-d)}{2} \int_{B_{R} \backslash B_{r_{*}}} \frac{u^{2}}{r^{3}}+F_{0}(v)-F(R, v) .
\end{aligned}
$$

Recall that

$$
\begin{aligned}
& -F(R, v) \\
& \quad=\Re\left(\int_{\partial B_{R}} \frac{r_{*}}{d-1}\left|v_{r}\right|^{2}-\frac{r_{*}}{d-1}\left|\nabla_{\partial B_{R}} v\right|^{2}+\frac{r_{*}}{2 R^{2}}|v|^{2}+\frac{r_{*}}{R} v_{r} \bar{v}+\frac{k^{2} r_{*}}{d-1}|v|^{2}\right) .
\end{aligned}
$$

Since $v$ satisfies the outgoing condition, it follows that ${ }^{\mathrm{n}}$ :

$$
\limsup _{R \rightarrow \infty}-F(R, v) \leq \frac{2 k^{2} r_{*}}{d-1} \limsup _{R \rightarrow \infty} \int_{\partial B_{R}}|v|^{2} .
$$

A combination of (5.28) and (5.34) yields

$$
\limsup _{R \rightarrow \infty}-F(R, v) \leq \frac{2 r \hat{\epsilon}^{2} k}{d-1} \int_{\Omega}|q|^{2}=C \epsilon^{2} \int_{\Omega}|q|^{2} .
$$

We have

$$
F_{0}(v)=\Re \int_{\partial B_{\tau}}-\frac{2 \tau}{d-1}\left|\partial_{n} v\right|^{2}+\frac{\tau}{d-1}|\nabla v|^{2}+\partial_{n} v \bar{v}-\frac{k^{2} \tau}{d-1}|v|^{2} .
$$

This implies

$$
F_{0}(v) \leq C\left(\int_{\partial B_{\tau}}|\nabla v|^{2}+k^{2} \int_{\partial B_{\tau}}|v|^{2}\right) .
$$

We derive from (5.29) that

$$
F_{0}(v) \leq C \int_{\Omega}|q|^{2} .
$$

${ }^{\mathrm{n}}$ For the details of the argument, see the one used to obtain (2.19) in Ref. 22. 
Combining (5.33), (5.35) and (5.38), we obtain

$$
\frac{1}{2(d-1)} \int_{B_{r_{*} \backslash B_{\tau}}}|\nabla v|^{2}+k^{2}|v|^{2} \leq C \int_{\Omega}|q|^{2}+\frac{r_{*}(3-d)}{2} \int_{B_{R} \backslash B_{r_{*}}} \frac{u^{2}}{r^{3}} .
$$

The proof for $d=3$ is complete. For $d=2$, it remains to absorb the second term on the right-hand side to the left-hand side. Without loss of generality, we may assume that $r_{*}$ is large enough. Then, the absorption can be done as in pp. 11-12 of Ref. 22. The details are left to the reader.

The following result will be used to obtain the estimate for $\mathbf{d}_{\ell}$.

Corollary 5.2. Let $d=2,3, k_{0}>0, r_{0}>0,0<\epsilon<1, k \geq k_{0}$, and $q \in L^{2}\left(\mathbb{R}^{d}\right)$ with $\operatorname{supp} q \subset \bar{\Omega}$. Let $v \in H_{\mathrm{loc}}^{1}\left(\mathbb{R}^{d}\right)$ be the unique outgoing solution to

$$
\Delta v+k^{2} v+i k \sigma_{\epsilon} v=q \quad \text { in } \mathbb{R}^{d} .
$$

Then, for any $K \subset \subset \mathbb{R}^{d} \backslash \bar{\Omega}$, there is a positive constant $C_{K}$ independent of $k, \epsilon$ and $q$ such that

$$
\|\nabla v\|_{L^{2}(K)}+k\|v\|_{L^{2}(K)} \leq C_{K} \hat{\epsilon} k^{2}\|q\|_{L^{2}(\Omega)} .
$$

Proof. The proof of this corollary is similar to that of Corollary 5.1. One only needs to use Lemma 5.5 (more precisely, estimate (5.26)) in place of Lemma 5.4. The details are left to the reader.

\subsection{Proof of Proposition 1.3}

Proposition 1.3 can now be proved in a similar way to Proposition 1.2. We only need to use Corollary 5.1 in place of Lemma 4.2 and Corollary 5.2 in place of Lemma 4.3. We present the proof here for the convenience of the reader. From the definition of $\mathbf{d}_{\ell}(3.14)$ and $\mathbf{e}_{\ell}(3.16)$, we have

$$
\left\|v^{\epsilon}-v_{\ell}^{a}\right\|_{H^{1}\left(B_{r} \backslash \Omega\right)} \leq\left\|\mathbf{d}_{\ell}\right\|_{H^{1}\left(B_{r} \backslash \Omega\right)}+\left\|\mathbf{e}_{\ell}\right\|_{H^{1}\left(B_{r} \backslash \Omega\right)} .
$$

Applying Corollary 5.1 and Lemma 3.4 (with $m=1$ ), we have, for $\ell=0,1$,

$$
\left\|\nabla \mathbf{e}_{\ell}\right\|_{L^{2}(K)}+k\left\|\mathbf{e}_{\ell}\right\|_{L^{2}(K)} \leq C k^{2}\left\|h_{\ell}\right\|_{L^{2}\left(\mathbb{R}^{d}\right)} \leq C k^{2 \ell+3} \hat{\epsilon}^{\ell+1}\|\mathbf{s}\|_{H^{2 \ell+1}\left(\mathbb{R}^{d}\right)} .
$$

Applying Corollary 5.2 and Lemma 3.3, we obtain, for $\ell=0,1,2$,

$$
\left\|\nabla \mathbf{d}_{\ell}\right\|_{L^{2}(K)}+k\left\|\mathbf{d}_{\ell}\right\|_{L^{2}(K)} \leq C \hat{\epsilon} k^{2}\left\|q_{\ell}\right\|_{L^{2}(\Omega)} \leq C k^{2 \ell+5} \hat{\epsilon}^{\ell}\|\mathbf{s}\|_{H^{2 \ell+3}\left(\mathbb{R}^{d}\right)} .
$$

This and Lemma 3.2 imply, for $\ell=0,1$,

$$
\begin{aligned}
\left\|\nabla \mathbf{d}_{\ell}\right\|_{L^{2}(K)}+k\left\|\mathbf{d}_{\ell}\right\|_{L^{2}(K)} \leq & \hat{\epsilon}^{\ell+1}\left(\left\|\nabla w_{e}^{\ell}\right\|_{L^{2}(K)}+k\left\|w_{e}^{\ell}\right\|_{L^{2}(K)}\right) \\
& +C k^{2 \ell+7} \hat{\epsilon}^{\ell+1}\|\mathbf{s}\|_{H^{2 \ell+5}\left(\mathbb{R}^{d}\right)} \\
\leq & C k^{2 \ell+7} \hat{\epsilon}^{\ell+1}\|\mathbf{s}\|_{H^{2 \ell+5}\left(\mathbb{R}^{d}\right)} .
\end{aligned}
$$

A combination of (5.39), (5.40) and (5.41) yields the conclusion. 


\section{Appendix. Proof of the Estimates for the Asymptotic Expansions}

\section{A.1. Proof of Lemma 3.1}

The conclusion of Lemma 3.1 follows from the definition of $w_{e}^{\ell}$ for $\ell=0,1,2$, and the standard regularity theory of elliptic equations. The details are left to the reader.

\section{A.2. Proof of Lemma 3.2}

Using the definition of $w_{e}^{0}$ and applying Lemma 5.2 for $r_{*}=R$, we have

$$
\left\|\nabla w_{e}^{0}\right\|_{L^{2}\left(B_{R} \backslash \Omega\right)}+k\left\|w_{e}^{0}\right\|_{L^{2}\left(B_{R} \backslash \Omega\right)} \leq C\|\mathbf{s}\|_{L^{2}\left(\mathbb{R}^{d} \backslash \Omega\right)} .
$$

Using the regularity theory of elliptic equations and applying Lemma 5.2, we have, for $m \geq 1$,

$$
\left\|\nabla w_{e}^{0}\right\|_{H^{m}\left(B_{R} \backslash \Omega\right)}+k\left\|w_{e}^{0}\right\|_{H^{m}\left(B_{R} \backslash \Omega\right)} \leq C k^{m}\|\mathbf{s}\|_{H^{m}\left(\mathbb{R}^{d} \backslash \Omega\right)},
$$

and hence by the trace theory, we obtain

$$
\left\|\partial_{n} w_{e}^{0}\right\|_{H^{m-1 / 2}(\Gamma)} \leq C\left\|w_{e}^{0}\right\|_{H^{m+1}\left(\mathbb{R}^{d} \backslash \Omega\right)} \leq C k^{m+1}\|\mathbf{s}\|_{H^{m+1}\left(\mathbb{R}^{d} \backslash \Omega\right)} .
$$

By (3.9) and (3.10), the conclusion follows from the definition of $w_{e}^{1}, w_{e}^{2}$ in (3.8), Lemma 5.2, and the standard regularity theory of elliptic equations.

\section{A.3. Proof of Lemma 3.3}

We only consider $k \geq k_{0}$. The other case follows similarly. From the definition of $q_{\ell}$, we have

$$
q_{\ell}(x)= \begin{cases}-\left[\Delta v_{i}^{\ell}(x)+k^{2} v_{i}^{\ell}(x)+\frac{i}{\hat{\epsilon}^{2}} v_{i}^{\ell}(x)\right] & \\ -\hat{\epsilon}^{\ell}\left[\Delta \varphi_{\hat{\epsilon}}(x)+k^{2} \varphi_{\hat{\epsilon}}(x)+\frac{i}{\hat{\epsilon}^{2}} \varphi_{\hat{\epsilon}}(x)\right] & \text { in } \Omega, \\ 0 & \text { in } \mathbb{R}^{d} \backslash \Omega .\end{cases}
$$

It follows that

$$
\left\|q_{\ell}\right\|_{L^{2}\left(\mathbb{R}^{d}\right)} \leq\left\|\Delta v_{i}^{\ell}+k^{2} v_{i}^{\ell}+\frac{i}{\hat{\epsilon}^{2}} v_{i}^{\ell}\right\|_{L^{2}(\Omega)}+\hat{\epsilon}^{\ell}\left\|\Delta \varphi_{\hat{\epsilon}}+k^{2} \varphi_{\hat{\epsilon}}+\frac{i}{\hat{\epsilon}^{2}} \varphi_{\hat{\epsilon}}\right\|_{L^{2}(\Omega)} .
$$

Since $\varphi_{\hat{\epsilon}}(x)=\nu \chi(\nu / \hat{\epsilon}) \partial_{n} w_{e}^{\ell}\left(x_{\Gamma}\right)$, we obtain

$$
\begin{aligned}
\hat{\epsilon}^{\ell}\left\|\Delta \varphi+k^{2} \varphi+\frac{i}{\hat{\epsilon}^{2}} \varphi\right\|_{L^{2}(\Omega)} \leq & C \hat{\epsilon}^{\ell}\left(\left\|\partial_{n} w_{e}^{\ell}\right\|_{H^{2}(\Gamma)}+k^{2}\left\|\partial_{n} w_{e}^{\ell}\right\|_{L^{2}(\Gamma)}\right) \\
& \times\left(\|\nu \chi(\nu / \hat{\epsilon})\|_{H^{2}(0, \infty)}+\frac{1}{\hat{\epsilon}^{2}}\|\nu \chi(\nu / \hat{\epsilon})\|_{L^{2}(0, \infty)}\right) .
\end{aligned}
$$


We have ${ }^{o}$ :

$$
\|\nu \chi(\nu / \hat{\epsilon})\|_{H^{2}(0, \infty)}+\frac{1}{\hat{\epsilon}^{2}}\|\nu \chi(\nu / \hat{\epsilon})\|_{L^{2}(0, \infty)} \leq C \hat{\epsilon}^{-1 / 2}
$$

Therefore,

$$
\hat{\epsilon}^{\ell}\left\|\Delta \varphi_{\hat{\epsilon}}+k^{2} \varphi_{\hat{\epsilon}}+\frac{i}{\hat{\epsilon}^{2}} \varphi_{\hat{\epsilon}}\right\|_{L^{2}(\Omega)} \leq C \hat{\epsilon}^{\ell-1 / 2}\left(\left\|\partial_{n} w_{e}^{\ell}\right\|_{H^{2}(\Gamma)}+k^{2}\left\|\partial_{n} w_{e}^{\ell}\right\|_{L^{2}(\Gamma)}\right) .
$$

We derive from Lemma 3.2 with $m=3$ that, for $\ell=0,1,2$,

$$
\hat{\epsilon}^{\ell}\left\|\Delta \varphi_{\hat{\epsilon}}+k^{2} \varphi_{\hat{\epsilon}}+\frac{i}{\hat{\epsilon}^{2}} \varphi_{\hat{\epsilon}}\right\|_{L^{2}(\Omega)} \leq C \hat{\epsilon}^{\ell-1 / 2} k^{2 \ell+3}\|\mathbf{s}\|_{H^{2 \ell+3}\left(\mathbb{R}^{d}\right)} .
$$

Using (3.4) and the definitions of $v_{i}^{1}$ and $v_{i}^{2}$, as in Ref. 12, we have

$$
\left\|\Delta v_{i}^{1}+k^{2} v_{i}^{1}+\frac{i}{\hat{\epsilon}^{2}} v_{i}^{1}\right\|_{L^{2}(\Omega)} \leq C\left(\left\|\mathcal{A}_{1} w_{i}^{1}\right\|_{L^{2}\left(\Gamma \times \mathbb{R}_{+}\right)}+\hat{\epsilon}^{-1}\left\|v_{i}^{1}\right\|_{H^{1}\left(\Gamma \times \mathbb{R}_{+}\right)}\right)
$$

and

$$
\begin{aligned}
& \left\|\Delta v_{i}^{2}+k^{2} v_{i}^{2}+\frac{i}{\hat{\epsilon}^{2}} v_{i}^{2}\right\|_{L^{2}(\Omega)} \\
& \quad \leq C\left(\hat{\epsilon}\left\|\mathcal{A}_{1} w_{i}^{2}+\mathcal{A}_{2} w_{i}^{1}+\mathcal{A}_{1} w_{i}^{2}\right\|_{L^{2}\left(\Gamma \times \mathbb{R}_{+}\right)}+\left\|v_{i}^{2}\right\|_{H^{1}\left(\Gamma \times \mathbb{R}_{+}\right)}\right) .
\end{aligned}
$$

Recall that:

$$
\begin{aligned}
& \mathcal{A}_{1}=2 \mathcal{H} \partial_{\eta}+6 \eta \mathcal{H}\left(\partial_{\eta}^{2}+i\right), \\
& \mathcal{A}_{2}=\Delta_{\Gamma}+k^{2}+2 \eta\left(\mathcal{G}+4 \mathcal{H}^{2}\right) \partial_{\eta}+3 \eta^{2}\left(\mathcal{G}+4 \mathcal{H}^{2}\right)\left(\partial_{\eta}^{2}+i\right) .
\end{aligned}
$$

Applying Lemma 3.2 with $(m=2$ and $\ell=0)$ and using (3.9), we obtain

$$
\left\|w_{i}^{1}\right\|_{L^{2}\left(\Gamma \times \mathbb{R}_{+}\right)}+\hat{\epsilon}^{-1}\left\|v_{i}^{1}\right\|_{H^{1}\left(\Gamma \times \mathbb{R}_{+}\right)} \leq C k^{2}\|\mathbf{s}\|_{H^{2}\left(\mathbb{R}^{d}\right)} .
$$

Applying Lemma 3.2 with $(m=3$ and $\ell=0)$ and $(m=1$ and $\ell=1)$ and using (3.10), we obtain

$$
\left\|w_{i}^{1}\right\|_{H^{2}\left(\Gamma \times \mathbb{R}_{+}\right)}+\left\|w_{i}^{2}\right\|_{L^{2}\left(\Gamma \times \mathbb{R}_{+}\right)} \leq C k^{3}\|\mathbf{s}\|_{H^{3}\left(\mathbb{R}^{d}\right)} .
$$

Moreover, applying Lemma 3.2 with $(m=2$ and $\ell=0,1)$,

$$
\hat{\epsilon}^{-1}\left\|v_{i}^{2}\right\|_{H^{1}\left(\Gamma \times \mathbb{R}_{+}\right)}=\left\|w_{i}^{1}+\hat{\epsilon} w_{i}^{2}\right\|_{H^{1}\left(\Gamma \times \mathbb{R}_{+}\right)} \leq C k^{4}\|\mathbf{s}\|_{H^{4}\left(\mathbb{R}^{d}\right)} .
$$

The conclusion now follows from (A.1), (A.3)-(A.8). We note here that for the case $\ell=0$, we use the fact that $v_{i}^{0} \equiv 0$.

\section{A.4. Proof of Lemma 3.4}

A calculation (see, e.g. (4.34) in Ref. 12) shows that $h_{0}=0, h_{1}=\frac{\hat{\epsilon}^{2}}{\alpha} \partial_{n} w_{e}^{1}$. The conclusion follows from Lemmas 3.1 and 3.2.

oThe scaling for the variable of $\chi$ gives us the optimal estimate in terms of $\hat{\epsilon}$. 


\section{Acknowledgment}

The first author thanks Patrick Joly and Houssem Haddar for introducing the subject. The first author was partially supported by NSF Grant DMS-1201370 and by the Alfred P. Sloan Foundation. The second author was partially supported by NSF Grant DMS-1212125.

\section{References}

1. X. Antoine, H. Barucq and L. Vernhet, High-frequency asymptotic analysis of a dissipative transmission problem resulting in generalized impedance boundary conditions, Asymptot. Anal. 26 (2001) 257-283.

2. X. Antoine and C. Besse, Construction, structure and asymptotic approximations of a microdifferential transparent boundary condition for the linear Schrödinger equation, J. Math. Pures Appl. 80 (2001) 701-738.

3. X. Antoine, C. Besse and P. Klein, Absorbing boundary conditions for the twodimensional Schrödinger equation with an exterior potential Part i: Construction and a priori estimates, Math. Models Methods Appl. Sci. 22 (2012) Article ID 1250026, $38 \mathrm{pp}$.

4. A. Bendali and K. Lemrabet, The effect of a thin coating on the scattering of a time-harmonic wave for the Helmholtz equation, SIAM J. Appl. Math. 56 (1996) 1664-1693.

5. T. A. Burton, Volterra Integral and Differential Equations, Mathematics in Science and Engineering, Vol. 167 (Academic Press, 1983).

6. G. Caloz, M. Dauge, E. Faou, V. Péron, On the influence of the geometry on skin effect in electromagnetism, Comput. Methods Appl. Mech. Engrg. 200 (2011) 1053-1068.

7. B. Delourme, H. Haddar and P. Joly, Approximate models for wave propagation across thin periodic interfaces, J. Math. Pures Appl. 98 (2012) 28-71.

8. B. Engquist and A. Majda, Absorbing boundary conditions for the numerical simulation of waves, Math. Comput. 31 (1977) 629-651.

9. E. Feiberg, On the propagation of radio waves along an imperfect surface. J. Phys. (Moscow) 8 (1944) 317-330.

10. I. M. Gelfand and G. E. Shilov, Generalized Functions, Vol. I: Properties and Operations (Academic Press, 1964). Translated by Eugene Saletan.

11. G. Grünberg, Suggestions for a theory of the coastal refraction, Phys. Rev. 63 (1943) 185.

12. H. Haddar, P. Joly and H.-M. Nguyen, Generalized impedance boundary conditions for scattering by strongly absorbing obstacles: The scalar case, Math. Models Methods Appl. Sci. 15 (2005) 1273-1300.

13. H. Haddar, P. Joly and H.-M. Nguyen, Generalized impedance boundary conditions for scattering by strongly absorbing obstacles: The case of Maxwell equations, Math. Models Methods Appl. Sci. 18 (2008) 1787-1827.

14. D. J. Hoppe and Y. Rahmat-Sami, Impedance Boundary Conditions in Electromagnetics (Taylor \& Francis, 1995).

15. L. Hörmander, $L^{p}$ estimates for (pluri-) subharmonic functions, Math. Scand. 20 (1967) 65-78.

16. P. Joly and S. Tordeux, Matching of asymptotic expansions for wave propagation in media with thin slots. I. The asymptotic expansion, Multiscale Model. Simulat. 5 (2006) 304-336.

17. M. A. Leontovich, Investigations on Radiowave Propagation, Part II (Printing House Academy of Science, 1949). 
18. R. C. MacCamy and E. Stephan, A skin effect approximation for eddy current problems, Arch. Rational Mech. Anal. 90 (1985) 87-98.

19. C. S. Morawetz and D. Ludwig, An inequality for the reduced wave operator and the justification of geometrical optics, Commun. Pure Appl. Math. 21 (1968) 187-203.

20. H.-M. Nguyen, Cloaking via change of variables for the Helmholtz equation in the whole space, Commun. Pure Appl. Math. 63 (2010) 1505-1524.

21. H.-M. Nguyen, Cloaking via change of variables for the Helmholtz equation in the whole space, Commun. Pure Appl. Math. 65 (2012) 155-186.

22. H.-M. Nguyen and M. S. Vogelius, Full range scattering estimates and their application to cloaking, Arch. Rational Mech. Anal. 203 (2012) 769-807.

23. H.-M. Nguyen and M. S. Vogelius, Approximate cloaking for the full wave equation via change of variables, SIAM J. Math. Anal. 44 (2012) 1894-1924.

24. H.-M. Nguyen and M. S. Vogelius, Approximate cloaking for the full wave equation via change of variables: The Lorentz model, submitted.

25. H.-M. Nguyen and L. V. Nguyen, Higher-order generalized impedance boundary conditions for time-dependent acoustic scattering by strongly absorbing obstacles, in preparation.

26. L. E. Payne and H. F. Weinberger, New bounds for solutions of second-order elliptic partial differential equations, Pacific J. Math. 8 (1958) 551-573.

27. B. Perthame and L. Vega, Morrey-Campanato estimates for Helmholtz equations, J. Funct. Anal. 164 (1999) 340-355.

28. F. Rellich, Darstellung der eigenwerte von $\Delta u+k u=0$ durch ein randintegral, Math. Z. 46 (1940) 635-636.

29. S. M. Rytov, Calcul du skin-effect par la méthode des perturbations, J. Phys. USSR 2 (1940) 233-242.

30. T. B. A. Senior and J. L. Volakis, Derivation and application of a class of generalized boundary conditions, IEEE Trans. Antennas and Propagation 37 (1989) 1566-1572.

31. A. N. Shchukin, Propagation of Radio Waves (Publishing House Svyazizdat, 1940). 